\title{
Recognition of human gastrointestinal cancer neoantigens by circulating PD-1+ lymphocytes
}

\author{
Alena Gros, ${ }^{1}$ Eric Tran, ${ }^{2,3}$ Maria R. Parkhurst, ${ }^{2}$ Sadia Ilyas, ${ }^{2}$ Anna Pasetto, ${ }^{4}$ Eric M. Groh, ${ }^{2}$ Paul F. Robbins, ${ }^{2}$ Rami Yossef, ${ }^{2}$ \\ Andrea Garcia-Garijo, ${ }^{1}$ Carlos A. Fajardo, ${ }^{1}$ Todd D. Prickett, ${ }^{2}$ Li Jia, ${ }^{2}$ Jared J. Gartner, ${ }^{2}$ Satyajit Ray, ${ }^{2}$ Lien Ngo, ${ }^{2}$ John R. Wunderllich, ${ }^{2}$ \\ James C. Yang, ${ }^{2}$ and Steven A. Rosenberg ${ }^{2}$
}

'Vall d'Hebron Institute of Oncology, Cellex Center, Barcelona, Spain. ${ }^{2}$ Surgery Branch, National Cancer Institute, NIH, Bethesda, Maryland, USA. ${ }^{3}$ Earle A. Chiles Research Institute, Robert W. Franz Cancer Center, Providence Cancer Institute, Portland, Oregon, USA. ${ }^{4}$ Department of Laboratory Medicine, Karolinska Institute, Stockholm, Sweden.

\begin{abstract}
Tumor-resident lymphocytes can mount a response against neoantigens expressed in microsatellite-stable gastrointestinal (GI) cancers, and adoptive transfer of neoantigen-specific lymphocytes has demonstrated antitumor activity in selected patients. However, whether peripheral blood could be used as an alternative minimally invasive source to identify lymphocytes targeting neoantigens in patients with $\mathrm{Gl}$ cancer with relatively low mutation burden is unclear. We used a personalized high-throughput screening strategy to investigate whether PD-1 expression in peripheral blood could be used to identify $\mathrm{CD8}^{+}$or $\mathrm{CD}^{+}$lymphocytes recognizing neoantigens identified by whole-exome sequencing in 7 patients with GI cancer. We found that neoantigen-specific lymphocytes were preferentially enriched in the CD8+PD-1+hi or CD4+PD-1+/hi subsets, but not in the corresponding bulk or PD-1- fractions. In 6 of 7 individuals analyzed we identified circulating CD8 ${ }^{+}$and CD4+ lymphocytes targeting 6 and 4 neoantigens, respectively. Moreover, neoantigen-reactive T cells and a T cell receptor (TCR) isolated from the CD8+PD-1+ subsets recognized autologous tumor, albeit at reduced levels, in 2 patients with available cell lines. These data demonstrate the existence of circulating T cells targeting neoantigens in $\mathrm{Cl}$ cancer patients and provide an approach to generate enriched populations of personalized neoantigen-specific lymphocytes and isolate TCRs that could be exploited therapeutically to treat cancer.
\end{abstract}

\section{Introduction}

Cancer neoantigens derived from mutated gene products can elicit $\mathrm{T}$ cell responses and render tumors susceptible to immune attack (1-3). Immune checkpoint inhibitors often display clinical activity in tumors with high mutation burden (4-8). However, most epithelial cancers, comprising $80 \%$ of all human malignancies, harbor a relatively low number of mutations $(9,10)$, and the frequency of tumor-reactive $\mathrm{CD} 8^{+}$lymphocytes has been shown to be variable and rare (11). Microsatellite-stable gastrointestinal (GI) cancers, including colorectum, pancreas, esophagus, bile duct, and gastric cancer, typically display a low frequency of somatic mutations $(9,10$, 12) and are largely refractory to treatment with immune checkpoint inhibitors. Despite their low mutation burden, $\mathrm{CD}^{+}$and/or $\mathrm{CD} 8^{+}$ neoepitope-specific responses can be detected in tumor-infiltrating lymphocytes (TILs) derived from GI tumors (13). More importantly, we reported that adoptive transfer of highly enriched $\mathrm{CD}^{+}$or $\mathrm{CD} 8^{+}$ mutation-specific lymphocyte populations targeting mutated ERB-

Conflict of interest: AG receives funding from Novartis, Roche, and EMD Serono, has received speaker honoraria from Roche, and has consulted for Achilles Therapeutics, Neon Therapeutics, and PACT Pharma. ET is on the scientific advisory board of PACT Pharma and Genocea Biosciences. AG and SAR are inventors on a patent application regarding the isolation of $T$ cells and $T$ cell receptors targeting neoantigens from peripheral blood (US Application 15/567,157).

Copyright: () 2019, American Society for Clinical Investigation.

Submitted: February 5, 2019; Accepted: August 13, 2019; Published: October 14, 2019.

Reference information: J Clin Invest. 2019;129(11):4992-5004.

https://doi.org/10.1172/JCI127967.
B2IP or KRAS neoantigens induced pronounced tumor regression in a patient with cholangiocarcinoma and a patient with colorectal cancer, respectively $(14,15)$. These case reports provide rationale for the development of personalized $\mathrm{T}$ cell therapies targeting neoantigens expressed by GI cancers.

The systematic detection and isolation of the endogenous neoantigen-specific T cells from GI cancer patients is largely restricted to tumor-resident $\mathrm{T}$ cells (13-15), which requires invasive methods and thus limits the applicability of personalized $\mathrm{T}$ cell therapies. A recent report demonstrated that, in 2 patients, neoantigen-reactive $\mathrm{T}$ cells present in the tumor were retrospectively found to exist in the peripheral blood memory compartment. The peripheral blood neoantigen-reactive cells were identified through 2 consecutive stimulations with candidate neoantigens to enrich for neoantigen-reactive $\mathrm{T}$ cell populations, and finally screening against individual peptides to assess neoantigen recognition (16). However, an approach to unbiasedly and prospectively identify neoantigen-specific lymphocytes from peripheral blood of GI cancer patients is still lacking. Detection of neoantigenreactive peripheral blood lymphocytes (PBLs) has been described in patients with epithelial cancers with relatively low mutation burden, such as ovarian cancer $(17,18)$. The strategies used to expand rare neoantigen-specific lymphocytes in peripheral blood require the screening of several thousand independent $\mathrm{T}$ cell cultures from a single patient (17) or rely on selection of mutated peptides using in silico prediction of peptide binding to MHC-I followed by several rounds of neoepitope-specific in vitro stimulation (18) - 
A

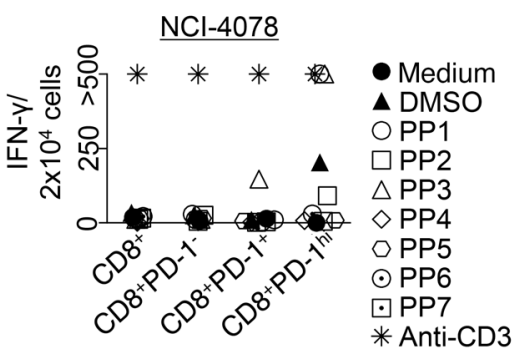

C
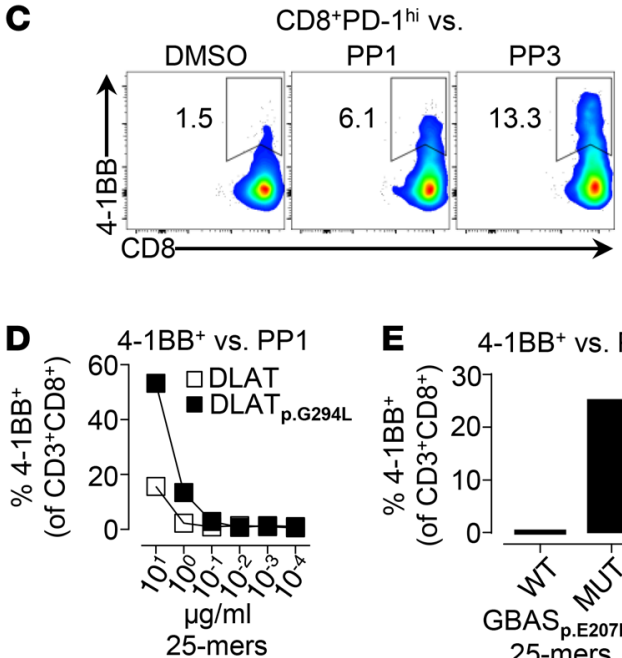

E $\quad 4-1 \mathrm{BB}^{+}$vs. PP3

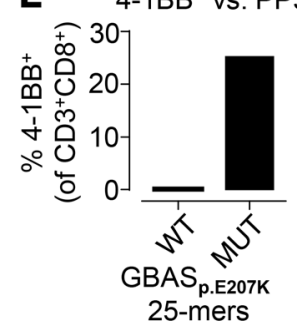

B

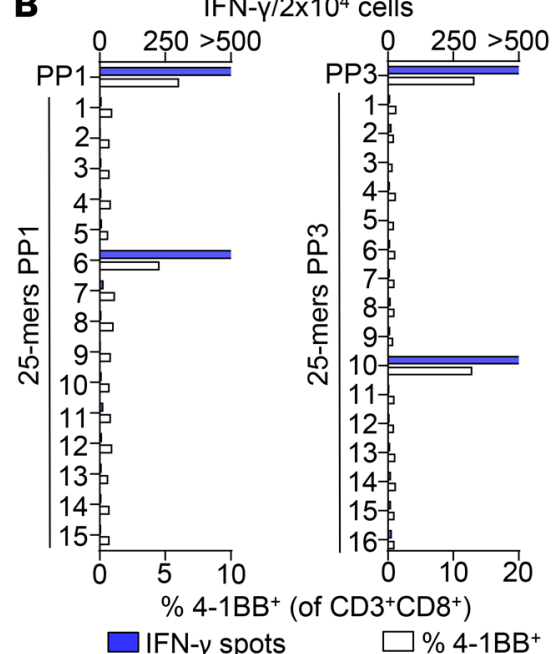

$\mathbf{F}$

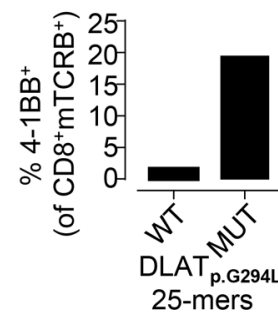

G

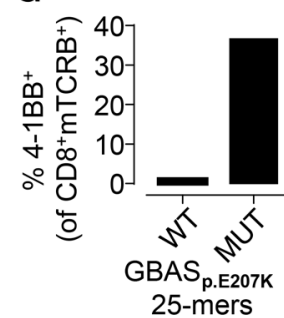

Figure 1. Identification of CD8+ neoantigen-specific lymphocytes in peripheral blood of a patient with gastroesophageal cancer (NCI-4078). (A) In vitroexpanded peripheral blood CD8+ lymphocyte subsets were cocultured with autologous DCs pulsed with DMSO or with the indicated peptide pools (PPs) containing the 25 -mers with the putative mutations identified by WES. T cell reactivity was measured by IFN- $\gamma$ ELISPOT assay. (B) Reactivity of peripheral blood CD8+PD- $1^{\text {hi }}$ cells to DCs pulsed with individual 25-mers from PP1 (left) or PP3 (right). IFN- $\gamma$ ELISPOT assay and flow cytometric analysis of 4-1BB upregulation on $C D 8^{+}$lymphocytes are plotted. (C) CD8 ${ }^{+} P D-1^{\text {hi }}$ T cells were cocultured with DCs pulsed with DMSO, PP1, or PP3, and the CD8 ${ }^{+}$T cells that upregulated 4-1BB against PP1 or PP3 were FACS purified and expanded. Representative plots show the percentage of $4-1 \mathrm{BB}^{+}$live $C D 3^{+} \mathrm{CD} 8^{+}$lymphocytes after coculture with the PPs specified. (D-C) PP1-reactive (D) or PP3-reactive (E) cells isolated in part C or PBLs transduced with candidate TCR1 (F) or TCR2 (G) were cocultured with autologous DCs pulsed with WT or mutated (MUT) DLAT ..C294L $_{\text {or }}$ CBAS $_{\text {p.E207K }}$ 25-mers, respectively. The constructed TCR expressed the mouse constant region, enabling its detection with antibodies specific for the mouse TCR $\beta$ constant region (mTCRB). Reactivity was measured by flow cytometric analysis of 4-1BB upregulation on $C D 8^{+}$cells ( $\mathbf{D}$ and $\mathbf{E}$ ) or $C D 8^{+} \mathrm{mTCRB}^{+}$lymphocytes $(\mathbf{F}$ and $\mathbf{G})$. The individual neoantigens recognized and the amino acid position and change are noted. " $>500$ " denotes greater than 500 spots. Experiments were performed without technical duplicates. Data from A-G are representative of at least 2 independent experiments.

methods that are cumbersome and can be ineffective for rare HLA molecules. Of potential concern regarding the therapeutic use of peripheral blood-derived neoantigen-reactive $\mathrm{T}$ cells is a recent study that demonstrated that in vitro-sensitized neoantigen-reactive $\mathrm{T}$ cells derived from the blood usually harbored $\mathrm{T}$ cells that targeted different neoantigens and displayed lower functional avidity than neoantigen-reactive T cells derived from TILs (18), suggesting that neoantigen-reactive $\mathrm{T}$ cells derived from the blood may not be the optimal source of antitumor lymphocytes.

Our previous data demonstrated that tumor-resident and circulating $\mathrm{CD} 8{ }^{+} \mathrm{PD}-1^{+}$lymphocytes in patients with melanoma represent an attractive source to identify and enrich for endogenous neoantigen-specific lymphocytes $(19,20)$. However, a recent report showed that $\mathrm{T}$ cell receptors isolated from $\mathrm{CD} 8^{+} \mathrm{T}$ cells infiltrating ovarian and colorectal cancers were rarely capable of recognizing autologous fresh tumor target cells even though they were frequently derived from PD-1-expressing $C D 8^{+}$lymphocytes (11), suggesting that the use of PD-1 expression to select for tumor-reactive and neoantigen-specific lymphocytes may be restricted to specific tumor types with high mutation burden, such as melanoma. In view of these findings, it is at present unclear whether or not PD-1 expression could be used to guide the enrichment of a systemic immune response targeting neoantigens in peripheral blood of patients with DNA mismatch repair-proficient GI cancer. Moreover, whether PD-1 can be used to enrich for circulating $\mathrm{CD}^{+}$neoantigen-specific lymphocytes is unknown.

\section{Results}

We investigated whether PD-1 expression on peripheral blood $\mathrm{CD} 8^{+}$and $\mathrm{CD} 4^{+}$lymphocytes could be used to identify circulating neoantigen-specific lymphocytes in 7 patients with advanced, refractory GI cancer. To this end, we performed tumor wholeexome sequencing (WES) from metastatic tumor deposits and identified between 32 and 151 nonsynonymous somatic mutations (NSMs) (Supplemental Table 1; supplemental material available online with this article; https://doi.org/10.1172/JCI127967DS1). Next, we synthesized 25-amino acid peptides (25-mers) and/or cloned minigenes encoding for all the candidate mutated 25-mer 
A

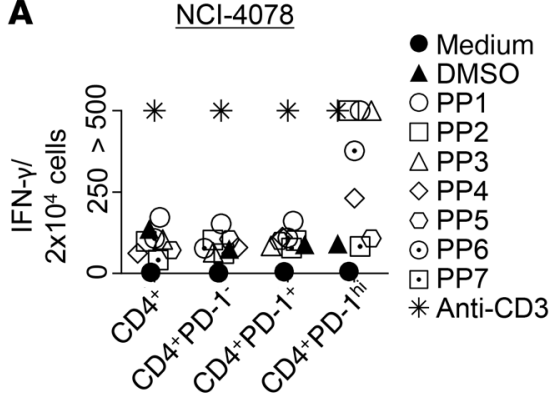

B

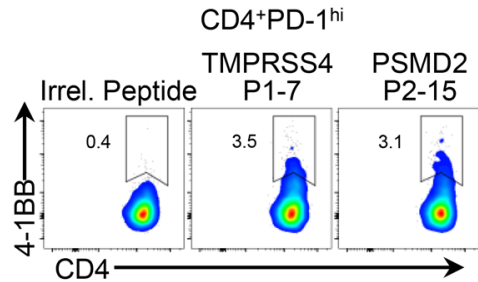

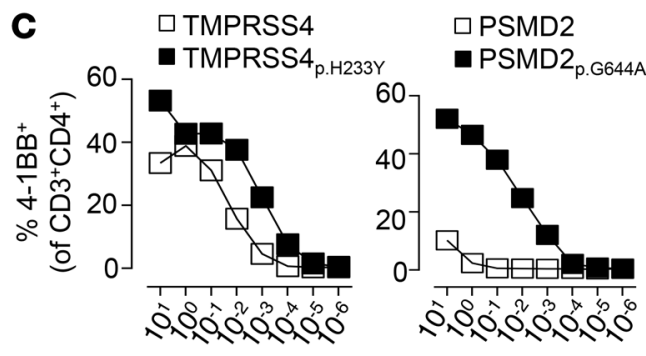

D

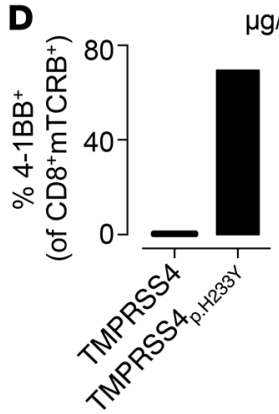

$\mu \mathrm{g} / \mathrm{ml} 25$-mers

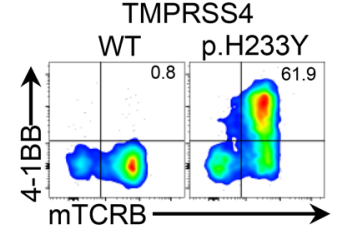

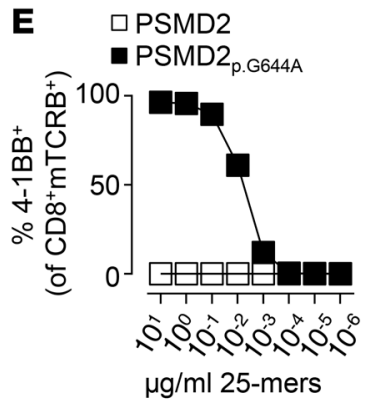

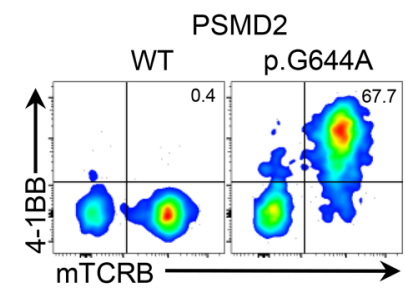

Figure 2. Detection of circulating CD4+ neoantigen-specific lymphocytes in a patient with gastroesophageal cancer (NCI-4078). (A) In vitro-expanded PBL subsets were cocultured with autologous DCs pulsed with DMSO or with the indicated PPs containing the putative mutations identified by WES. T cell reactivity was measured the next day by IFN- $\gamma$ ELISPOT assay. (B) Reactivity of peripheral blood CD4+PD- - $^{\text {hi }}$ cells to DCs pulsed with an irrelevant peptide or peptides P1-7 and P2-15. Representative plots display the percentage of 4-1BB expression on live CD3+CD4+ lymphocytes. (C) P1-7- and P2-15-reactive cells isolated in B and expanded were cocultured with DCs pulsed with decreasing concentrations of TMPRSS4 ${ }_{\mathrm{D} . \mathrm{H} 233 \mathrm{Y}}$ and PSMD2 $2_{\mathrm{p} .644 \mathrm{~A}}$ WT and mutated 25 -mers. Flow cytometric analysis of 4-1BB upregulation on $\mathrm{CD3}^{+} \mathrm{CD} 4^{+}$cells is plotted. ( $\mathbf{D}$ and $\mathbf{E}$ ) Reactivity of gene-engineered PBLs with dominant TMPRSS4 ${ }_{\text {p.H233Y }}$ or $^{-}$ PSMD2 $_{\text {p.6644A }}$-specific candidate TCR- $\alpha / \beta$ pairs from Table 1 to autologous DCs pulsed with WT and mutated TMPRSS4 ${ }_{\text {p.H233Y }}$ (D) and PSMD2 $2_{\text {p.6644A }}$ (E) $25_{-m}$-mers Reactivity was measured by flow cytometric analysis of 4-1BB upregulation on CD8 ${ }^{+} m$ TCRB $^{+}$lymphocytes, and representative plots are shown. The individual neoantigens recognized and the amino acid position and change are noted. " $>500$ " denotes greater than 500 spots per $2 \times 10^{4}$ cells. Experiments were performed without technical duplicates. Data from A-E are representative of at least 2 independent experiments.

epitopes identified with the mutated amino acid in position 13 of the peptide/minigene, as previously described $(3,13)$. For screening, peptides were grouped into peptide pools (PPs), each containing up to 17 peptides, and PPs were pulsed onto autologous antigen-presenting cells (APCs). Alternatively, DNA tandem minigene (TMG) constructs, each encoding up to 17 concatenated minigenes, were used to generate in vitro-transcribed RNA, which was electroporated into autologous APCs. In parallel, we isolated $\mathrm{CD} 8^{+}$and $\mathrm{CD} 4^{+} \mathrm{T}$ cells from peripheral blood based on PD-1 expression into PD-1 ${ }^{-}$PD- $-1^{+}$, and PD- $1^{\text {hi }}$ (defined as the top $20 \%$ of PD-1-expressing cells) and expanded them in vitro. The mean percentage of PD-1-expressing lymphocytes in the 7 patients studied in detail was $4.81 \% \pm 2.45 \%$ and $3.87 \% \pm 1.97 \%$ in peripheral blood $\mathrm{CD} 8^{+}$or $\mathrm{CD} 4^{+} \mathrm{T}$ cells, respectively (Supplemental Table 1). Peptide-pulsed APCs or APCs electroporated with in vitro-transcribed TMG RNA were used as targets in coculture assays to screen the expanded lymphocyte subsets for neoantigen recognition. This strategy allows for potential processing and presentation of all the mutated candidate antigens identified by all autologous MHC-I and MHC-II molecules. Finally, T cell responses were evaluated by measurement of IFN- $\gamma$ secretion using an IFN- $\gamma$ ELISPOT and upregulation of costimulatory receptors $4-1 \mathrm{BB}$ or OX40, as these provide complementary functional measures of $\mathrm{T}$ cell activation $(14,21)$.

Patient NCI-4078 had refractory, metastatic gastroesophageal cancer, and WES revealed 117 NSMs in this patient's tumor. We synthesized 104 unique 25-mers representing all the transcribed putative neoantigens, each 25 -mer containing the putative mutated amino acid flanked by wild-type amino acids of the protein, and grouped the 25-mers into 7 independent PPs (PP1-PP7). The CD8 ${ }^{+} \mathrm{PD}-1^{+}$lymphocytes displayed low reactivity against $\mathrm{PP} 3$, and the circulating $\mathrm{CD} 8^{+} \mathrm{PD}-1^{\text {hi }}$ lymphocytes 
Table 1. Single-cell TCR sequencing of CD4+4-1BB+ sorted lymphocytes derived from the blood of patient NCI-4078

\begin{tabular}{|c|c|c|}
\hline \multirow{2}{*}{$\begin{array}{l}\text { Population sorted } \\
\text { CD4+PD-1hin-1BB }{ }^{+} \text {vs. PP1 }\end{array}$} & Candidate neoantigen targeted & $\mathrm{CDR}_{\alpha}$ amino acid sequence \\
\hline & TMPRSS4 $_{\text {p. } 1233 \mathrm{Y}}$ & CACSGGSEKLVF \\
\hline $\mathrm{CD}^{+} \mathrm{PD}-1^{\mathrm{hi}} 4-1 \mathrm{BB}^{+}$vs. PP2 & PSMD2 $_{0.644 A}$ & $\begin{array}{l}\text { CAVSENNYGQNFVF } \\
\text { CAVVSGTYKYIF }\end{array}$ \\
\hline
\end{tabular}

CDR3 $_{\beta}$ amino acid sequence
CASSSSCAFQPQHF
CASRGDIPSDTQYF
CASSVGLAGAYEQFF

Number of times detected
13
1
8

TCR- $\alpha \beta$ pairs identified by single-cell TCR sequencing from selected $4-1 \mathrm{BB}^{+}$cells following coculture of PP1- or PP2-reactive cells with autologous DCs pulsed with TMPRSS4 $4_{\text {p.H233Y }}$ and PSMD2 ${ }_{\text {p.G644A }}$. CDR3 amino acid sequences of the TRA and TRB loci and number of times each TCR- $\alpha / \beta$ pair was detected are shown.

recognized neoantigens within PP1 and PP3 (Figure 1A), whereas no activity was seen in the patient's peripheral blood $\mathrm{CD}^{+}$or CD $8^{+} \mathrm{PD}-1^{-} \mathrm{T}$ cells. Further analysis of the specific neoantigens targeted showed that approximately $6 \%$ of the in vitro-expanded circulating $\mathrm{CD} 8^{+} \mathrm{PD}-\mathrm{1}^{\text {hi }}$ lymphocytes (Figure $1 \mathrm{~B}$ ) targeted a neoepitope containing a mutated amino acid in the DLAT protein, referred to as DLAT $\mathrm{p}_{\text {.G294L }}$, which denotes a change from a glycine to a leucine at position 294, while an additional $11 \%$ of the in vitro-expanded circulating $\mathrm{CD} 8^{+} \mathrm{PD}-\mathrm{1}^{\text {hi }}$ lymphocytes recognized $\mathrm{GBAS}_{\mathrm{p} . \mathrm{E207K}}$. Given the relatively low frequency and the polyclonal nature of the circulating $\mathrm{CD} 8^{+} \mathrm{PD}-1^{\text {hi }}$ subset, we next enriched for DLAT $_{\text {p.G294L }}$ - and GBAS ${ }_{\text {p.E207K }}$-specific lymphocytes by selecting the $4-1 \mathrm{BB}^{+}$lymphocytes after coculturing the circulating $\mathrm{CD}^{+}$ PD-1 ${ }^{\text {hi }}$-derived lymphocytes with DCs pulsed with DLAT $_{\text {p.G294L }}$ or GBAS $_{\text {p.E207K }} 25$-mers and expanding them in vitro (Figure 1C). The resulting $\mathrm{DLAT}_{\mathrm{p} . \mathrm{G} 294 \mathrm{~L}}{ }^{-}$or $\mathrm{GBAS}_{\mathrm{p} . \mathrm{E} 207 \mathrm{~K}}$-enriched lymphocytes displayed preferential recognition of the mutated 25-mers, as compared with the WT counterparts (Figure 1, D and E), demonstrating the existence of a circulating $\mathrm{CD}^{+}$response against 2 neoantigens in this patient. Moreover, neoantigen-specific $\mathrm{CD}^{+}$lymphocytes were preferentially enriched in the peripheral blood $\mathrm{CD} 8^{+} \mathrm{PD}-1^{+}$ and $\mathrm{CD} 8^{+} \mathrm{PD}-\mathrm{1}^{\mathrm{hi}}$ subsets.

In addition to generating enriched populations of $\mathrm{CD} 8^{+}$neoantigen-specific lymphocytes from peripheral blood, we construct- ed and tested $\mathrm{T}$ cell receptors (TCRs) for neoantigen recognition following 2 approaches outlined in Supplemental Figure 1, which relied on either bulk TCR or single-cell TCR sequencing to identify candidate neoantigen-specific TCR- $\alpha \beta$ pairs. Deep sequencing of the variable V-J or V-D-J region of the TCR- $\alpha$ and TCR- $\beta$ loci (TRA and TRB, respectively) of the DLAT ${ }_{\mathrm{p} . \mathrm{G} 294 \mathrm{~L}}$-enriched lymphocytes, for example, revealed multiple dominant TRB and TRA clonotypes. We constructed TCRs by pairing the 2 most dominant TRA and TRB pairs and subcloned them into retroviral vectors that were used to transduce autologous PBLs. The TCR constructed using the most dominant TRA and TRB CDR3 sequences (CDR3 ${ }_{\alpha}$ and $C D R 3_{\beta}$, respectively) displayed specific recognition of DLAT $_{\text {p.G294L }}$ (Figure $1 \mathrm{~F}$ and Supplemental Table 2), as shown by the upregulation of $4-1 \mathrm{BB}$ on the transduced cells following coculture with autologous DCs pulsed with DLAT ${ }_{\text {p.G294L }} 25$-mer. We also performed single-cell sequencing of the CDR3 ${ }_{\alpha}$ and $\mathrm{CDR} 3_{\beta}$ regions of the $4-1 \mathrm{BB}^{+}$cells following coculture of $\mathrm{CD} 8^{+} \mathrm{PD}-1^{\text {hi }}$ cells with GBAS $_{\text {p.E207K }} 25$-mer. We detected 2 candidate TCR- $\alpha \beta$ pairs, which shared the same $\mathrm{CDR} 3_{\beta}$ sequence. Both TCRs were subcloned into retroviral vectors, used to transduce autologous PBLs, and one of them recognized GBAS ${ }_{\text {p.E207K }}$ 25-mer, but not the WT counterpart (Figure $1 \mathrm{G}$ and Supplemental Table 2). Thus, neoantigen-specific TCRs targeting DLAT p.G294L or GBAS ${ }_{\text {p.E207K }}$ were isolated from the circulating CD8 ${ }^{+} \mathrm{PD}-1^{\text {hi }}$-expressing lymphocytes in patient NCI-

Table 2. Neoantigens targeted by circulating lymphocytes from patients with GI cancers

\begin{tabular}{|c|c|c|c|c|c|c|}
\hline Patient ID & Tumor type & $\begin{array}{c}\text { Number of somatic } \\
\text { NSMs }^{\mathrm{A}, \mathrm{B}}\end{array}$ & $\begin{array}{c}\text { Number of mutated epitopes } \\
\text { evaluated }^{\mathrm{C}}\end{array}$ & $\begin{array}{l}\text { Peripheral blood subset with } \\
\text { neoantigen reactivity }\end{array}$ & $\begin{array}{l}\text { Mutated protein } \\
\text { recognized }\end{array}$ & $\begin{array}{l}\text { Amino acid position } \\
\text { and change }\end{array}$ \\
\hline \multirow[t]{3}{*}{$\mathrm{NCl}-4078$} & Gastro-esophageal & 117 & 104 & $\mathrm{CD} 8^{+} \mathrm{PD}-1^{\mathrm{hi}}$ & DLAT & p.G294L \\
\hline & & & & $\mathrm{CD}^{+} \mathrm{PD}-1^{\mathrm{hi}}$ & PSMD2 & p.G644A \\
\hline & & & & $\mathrm{CD}^{+} \mathrm{PD}-1^{\mathrm{hi}}$ & TMPRSS4 & p.H233Y \\
\hline $\mathrm{NCl}-4095^{\mathrm{E}}$ & Colon & $92,78,112^{\mathrm{F}}$ & 61 & $\mathrm{CD} 8^{+} \mathrm{PD}-1^{+}$ & KRAS & p.G12D \\
\hline $\mathrm{NCl}-4114$ & Pancreatic & 45 & 46 & None detected & & \\
\hline \multirow[t]{2}{*}{ NCl-4166 } & Colon & 74 & 74 & CD8+PD-1+/hi & NPLOC4 & p. $1312 \mathrm{~V}$ \\
\hline & & & & $C D 4^{+} P D-1^{\text {hi }}$ & SUN1 & p.A8T/p.A177T \\
\hline NCI-4177 & Pancreatic & 43 & 45 & CD8 $8^{+} P D-1^{\mathrm{hi}}$ & MMP14 & p.R158C \\
\hline
\end{tabular}

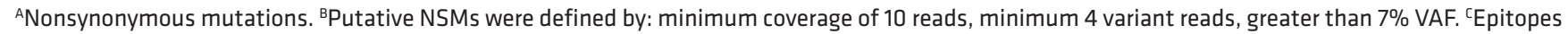
were selected for screening as explained in detail in Methods. ${ }^{\mathrm{D}}$ Tumor from NCI-MM was sequenced and analyzed externally, and the specific filters used to identify the NSMs are unknown. EPatient 4095 was previously reported in ref. 15. F,GThe number of mutations from 3 independent metastatic tumor deposits from patient $\mathrm{NCl}-4095$ and 2 independent metastases from patient $\mathrm{NCl}-4110$ sequenced in parallel is shown. 
A

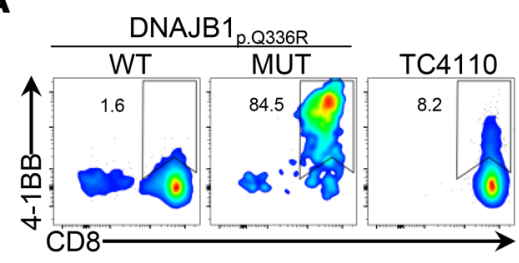

B

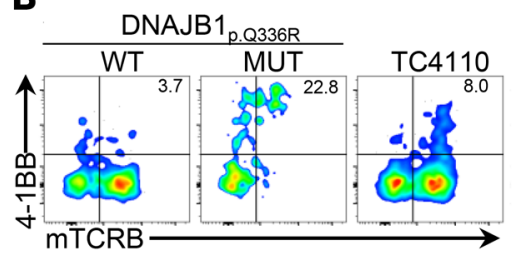

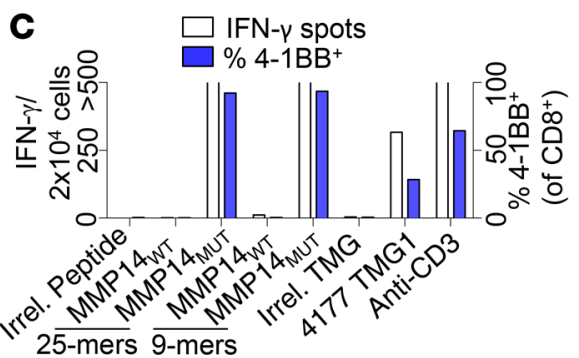

D

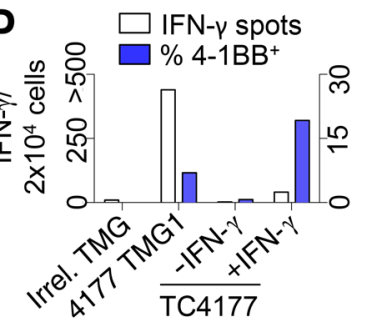

E

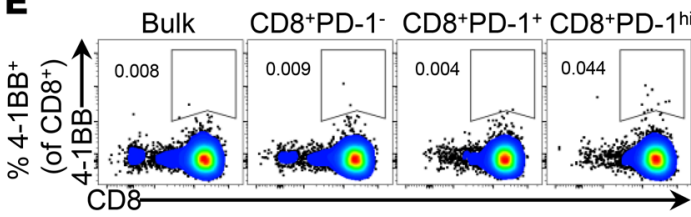

$\mathbf{F}$

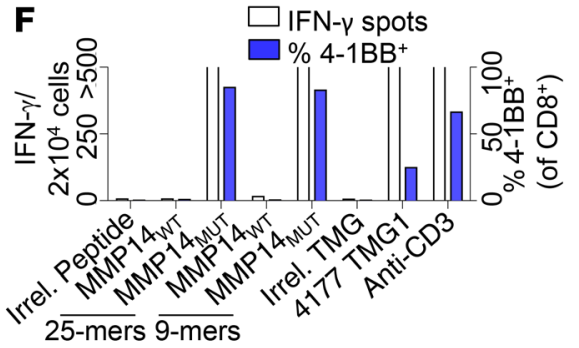

G

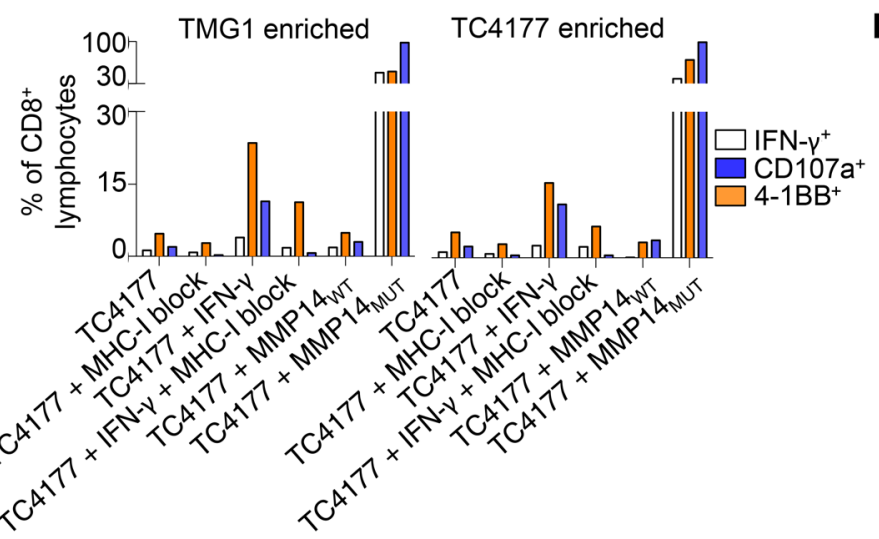

H

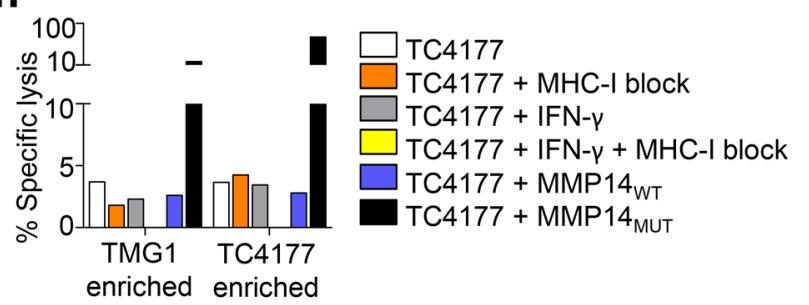

Figure 3. Autologous tumor recognition by circulating neoantigen-specific lymphocytes or TCR-transduced cells from NCI-4110 and NCI-4177. (A and B) Reactivity of DNAJB1-reactive lymphocytes (A) or PBLs transduced with a DNAJB1-specific TCR isolated from NCI-4110 (see Supplemental Figure 5) (B) following coculture with APCs pulsed with WT or MUT DNAJB1 25-mers, or with TC4110. Percentage of transduced cells was quantified using an antibody targeting the mouse TCR $\beta$ constant region (mTCRB). The percentage of live $C D 3^{+}$cells expressing 4-1BB is shown. (C and D) Reactivity of TMC1-reactive

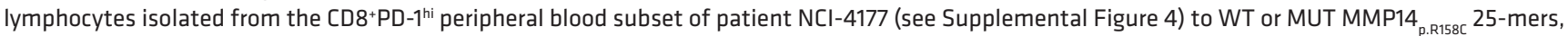
irrelevant TMG RNA, or 4177-TMG1 RNA, encoding MMP14 ${ }_{\text {p.R158c }}$ (C), or to irrelevant TMG RNA, 4177-TMG1 RNA, or TC4177 (D). IFN- $\gamma$ ELISPOT and flow cytometric analysis of 4-1BB expression on $C D 8^{+}$cells are plotted. (E) 4-1BB expression on the $T$ cell subsets following coculture with the autologous tumor. The percentage of live, $\mathrm{CD}^{+}$lymphocytes expressing 4-1BB is shown. (F) IFN- $\gamma$ ELISPOT and flow cytometric analysis of 4-1BB expression on in vitro-expanded TC4177-reactive CD8 ${ }^{+}$PD- $1^{\text {hi }}$ T cells isolated in E following coculture with APCs pulsed with WT and MUT MMP14 ${ }_{\text {pR158c }} 25$-mers or 9-mers, or electroporated with encoding irrelevant TMG RNA or 4177-TMG1 RNA. (G and $\mathbf{H}$ ) Tumor recognition (intracellular IFN- $\gamma$, cell surface CD107a, and 4-1BB) (G) and autologous tumor lysis $(\mathbf{H})$ by MMP14 ${ }_{\text {p.R158c }}$-reactive lymphocytes generated through enrichment of 4177-TMC1-reactive lymphocytes shown in $\mathbf{C}$ and $\mathbf{D}$ (left panel of $\mathbf{G}$ ) or enrichment of TC4177-reactive lymphocytes shown in $\mathbf{F}$ (right panel of $\mathbf{G}$ ) in the treatment conditions specified. The mean percentage lysis of technical duplicates is plotted. ">500" denotes greater than 500 spots per $2 \times 10^{4}$ cells. Except in $\mathbf{H}$, experiments were performed without duplicates. Data from A-H are representative of at least 2 independent experiments.

4078, demonstrating that this approach can be harnessed to isolate personalized neoantigen-specific TCRs that could be used to treat cancer.

We next attempted to identify circulating $\mathrm{CD} 4^{+}$neoantigen-specific responses in patient $\mathrm{NCI}-4078$. The screening of the $\mathrm{CD} 4^{+} \mathrm{PBL}$ subsets revealed that the $\mathrm{CD} 4^{+} \mathrm{PD}-1^{\text {hi }}-$ derived lymphocytes, but not the $\mathrm{CD} 4^{+}, \mathrm{CD} 4^{+} \mathrm{PD}-1^{-}$, or $\mathrm{CD} 4^{+} \mathrm{PD}-1^{+}$cells, recognized mutated 25-mers included in the PPs identified by WES (Figure 2A). Further analysis showed that this population displayed reactivity against peptides P1-7 and P2-15, corresponding to mutated TMPRSS4 $4_{\text {.. } 2333 \mathrm{Y}}$ and PSMD $2_{\mathrm{p} . \mathrm{G} 644 \mathrm{~A}}$ included in PP1 and PP2, respectively (Figure 2B).
The CD4 ${ }^{+} \mathrm{PD}-1^{\text {hi }}$ lymphocytes capable of expressing 4-1BB following coculture with TMPRSS $4_{\mathrm{p} . \mathrm{H} 233 \mathrm{Y}}$ and PSMD2 $2_{\mathrm{p} . \mathrm{G} 644 \mathrm{~A}}$ (Figure 2B) were expanded in vitro to generate enriched populations of neoantigen-reactive cells and to identify putative neoantigen-reactive TCR- $\alpha \beta$ pairs. The resulting TMPRSS4 $4_{\mathrm{p} . \mathrm{Hz} 23 \mathrm{Y}}$-enriched lymphocytes displayed marginal selective reactivity against the mutated antigen compared with the WT peptide, while the PSMD2 ${ }_{\mathrm{p} .6644 \mathrm{~A}}$-enriched lymphocytes displayed specific recognition of the mutated epitope (Figure 2C). Single-cell TCR sequencing of the TMPRSS $4_{\text {p.H233Y }}$ and PSMD $2_{\mathrm{p} .6444 \mathrm{~A}}$-reactive $4-1 \mathrm{BB}^{+}$lymphocytes identified 1 dominant TCR- $\alpha \beta$ pair for each of the TMPRSS $4_{\mathrm{p} . \mathrm{H} 233 \mathrm{Y}}$ and PSMD $2_{\mathrm{p} . \mathrm{G} 644 \mathrm{~A}}$ pop- 
ulations (Table 1). Both TCRs demonstrated neoantigen-specific recognition when transduced into PBLs, as shown by the upregulation of 4-1BB within the transduced $\mathrm{T}$ cell population following coculture with autologous APCs pulsed with TMPRSS $4_{\text {p.H233Y }}$ and PSMD $2_{\text {p.G644A }}$ mutated 25-mers, but not with the WT antigen (Figure 2, D and E, respectively). As shown, neoantigen recognition was CD4 coreceptor independent, since transduced CD8 $8^{+}$lymphocytes expressed costimulatory receptor 4-1BB in response to the neoantigen. Notably, our screening approach identified 2 patient-specific $\mathrm{CD} 4^{+}$neoantigen-specific TCRs, and selection of $\mathrm{CD} 4^{+} \mathrm{PD}-1^{\text {hi }}$ circulating lymphocytes was required to detect the endogenous $\mathrm{CD}^{+}$ response to neoantigens.

We used a similar strategy to evaluate the presence of $\mathrm{CD}^{+}$ and $\mathrm{CD}^{+}$neoantigen-specific lymphocytes in peripheral blood of 6 additional patients with metastatic pancreatic, colorectal, and bile duct cancers (Supplemental Figures 2-6). A summary of the specific neoantigens targeted for each of the patients studied and the specific $\mathrm{T}$ cell subsets displaying recognition of each neoantigen is shown in Table 2. We detected neoantigen-specific lymphocytes in 6 of 7 patients targeting a total of 10 neoantigens. In patient NCI-4095, a patient with metastatic colorectal cancer, the circulating CD $8^{+} \mathrm{PD}-1^{+}$ subset isolated from the pheresis obtained before adoptive cell transfer contained T cells targeting KRAS $_{\text {p.G12D }}$ (Supplemental Figure 3), expressed in $45 \%$ of pancreatic (22) and $13 \%$ of colorectal cancer (23). Interestingly, this patient experienced tumor regression following treatment with $\mathrm{KRAS}_{\mathrm{p} . \mathrm{G} 12 \mathrm{D}}$-specific TILs (15). The remaining neoantigens identified using this approach were unique to each patient's tumor and had not previously been reported. Furthermore, consistent with the representative patient shown, neoantigenreactive cells were preferentially enriched in the $\mathrm{CD} 4^{+} \mathrm{PD}-\mathrm{1}^{\mathrm{hi}}$ and/ or the $\mathrm{CD} 8^{+} \mathrm{PD}-1^{+}$and $\mathrm{CD} 8^{+} \mathrm{PD}-1^{\text {hi }}$ subsets, but not detected in the PD-1 ${ }^{-}$counterparts (Table 2). The frequency of neoantigen-reactive lymphocytes within the ex vivo-expanded CD $4^{+} \mathrm{PD}-1^{\mathrm{hi}}, \mathrm{CD} 8^{+} \mathrm{PD}-1^{+}$, and $C D 8^{+} \mathrm{PD}-1^{\text {hi }}$ populations as determined by $4-1 \mathrm{BB}$ upregulation ranged from $0.14 \%$ up to $11 \%$. In addition to the 4 neoantigen-specific TCRs identified in NCI-4078, 2 neoantigen-specific TCRs were isolated through single-cell TCR sequencing from NCI-4095 and NCI-4110 targeting KRAS $_{\text {p.G12D }}$ and DNAJB1 ${ }_{\text {p.Q336R }}$, respectively (Supplemental Figures 3 and 5), and these are summarized in Supplemental Table 2. Four of the neoantigens identified were targeted by circulating $\mathrm{CD} 4^{+} \mathrm{PD}-1^{\text {hi }}$ lymphocytes, demonstrating that $\mathrm{CD} 4^{+}$ neoantigen-reactive lymphocytes can upregulate PD-1, and selection of the peripheral blood CD $4^{+} \mathrm{PD}-1^{\text {hi }}$ subset can enrich for $\mathrm{CD} 4^{+}$ neoantigen-reactive lymphocytes.

To further explore whether the neoantigen-specific TCRs isolated from peripheral blood could be clinically relevant, we interrogated their presence within the corresponding metastatic tumor deposits from patients NCI-4078, NCI-4095, and NCI-4110. The presence of these cells in the tumor could suggest that these $\mathrm{T}$ cells expanded locally in response to their cognate antigen. The 6 TCRs targeting neoantigens identified in the blood through $\mathrm{CD} 8^{+} \mathrm{PD}-1^{+}, \mathrm{CD} 8^{+} \mathrm{PD}-1^{\mathrm{hi}}$, or $\mathrm{CD} 4^{+} \mathrm{PD}-1^{\text {hi }}$ lymphocyte selection either were found at a very low frequency or were undetectable in bulk peripheral blood by TCRB deep sequencing (Supplemental Table 3). However, all but one neoantigen-specific TCR were found to be enriched at the tumor site. In patient NCI-4078, all 4 circulating, mutation-specific TCRs identified were detected in the corresponding fresh tumor. Moreover, 3 of the 4 neoantigen-specific TCR clonotypes recognizing DLAT ${ }_{\text {p.G294L }}$, GBAS $_{\text {p.E207K }}$, and TMPRSS $4_{\text {p.H233Y }}$ were detected at a higher frequency in the tumor-resident $\mathrm{CD} 8^{+} \mathrm{PD}-1^{+}$or $\mathrm{CD} 4^{+} \mathrm{PD}-1^{+}$lymphocytes compared with their PD-1 $1^{-}$counterparts. The PSMD2-specific TCR was not detected in the bulk $\mathrm{CD} 4^{+}, \mathrm{CD} 4^{+} \mathrm{PD}-1^{+}$, or $\mathrm{CD} 4^{+} \mathrm{PD}-1^{-}$subsets. For patient NCI-4095, the KRAS $_{\text {p.G12D }}$-specific TCR- $\beta$ clonotype that we identified in the peripheral blood $\mathrm{CD} 8^{+} \mathrm{PD}-1^{+}$subset ranked among the 2 most frequent TCRB clonotypes in 3 independent tumor biopsies, representing 2.68\%, 2.81\%, and $2.71 \%$ of all $\mathrm{T}$ cells (Supplemental Table 3). The preferential detection of the peripheral blood neoantigen-specific TCRs within the tumor and the dominance of the $\mathrm{KRAS}_{\mathrm{p.G12D}}$ TCR clonotype are consistent with local antigen-specific clonal expansion and suggested that the peripheral blood PD-1/hi-derived neoantigen-specific TCRs identified could be relevant for patient treatment. Only 1 of the 6 peripheral blood neoantigen-specific TCRs identified, targeting DNAJB1 ${ }_{\mathrm{p} . \mathrm{Q} 336 \mathrm{R}}$ in patient NCI-4110, was not detected in either of two 4110 metastatic deposits sequenced.

More importantly, we generated a cholangiocarcinoma cell line (TC4110) and a pancreatic cancer cell line (TC4177) from patients NCI-4110 and NCI-4177, respectively, which enabled us to directly interrogate whether neoantigen-specific lymphocytes identified in blood could recognize autologous tumor cells. DNAJB1 ${ }_{\text {p.0336R }}$-specific $\mathrm{T}$ cells enriched from the peripheral blood CD8 ${ }^{+} \mathrm{PD}-1^{+}$subset of patient NCI-4110 (Figure 3A and Supplemental Figure 5) as well as PBMCs transduced with the peripheral blood-derived neoantigen-specific TCR targeting DNAJB1 ${ }_{\text {p.Q336R }}$ (Figure 3B and Supplemental Figure 5F) displayed autologous TC4110 recognition, as evidenced by the upregulation of 4-1BB when cocultured with TC4110 (Figure 3, A and B). Furthermore, MMP14 ${ }_{\mathrm{p} . \mathrm{R} 158 \mathrm{C}}$-specific T lymphocytes isolated from the peripheral blood CD $8{ }^{+} \mathrm{PD}-1^{\text {hi }}$ subset of patient NCI-4177 (Figure 3C and Supplemental Figure 4) recognized TC4177 following pretreatment of the cell line with IFN- $\gamma$ (Figure 3D), as demonstrated by the IFN- $\gamma$ ELISPOT and by upregulation of 4-1BB expression after coincubation of this effector T cell population with the IFN- $\gamma$-treated cell line. Additionally, isolation and expansion of a small fraction (0.044\%) of TC4177-reactive $4-1 \mathrm{BB}^{+}$cells derived from the circulating CD8 ${ }^{+}$PD $-1^{\text {hi }}$ subset of patient NCI-4177 (Figure $3 \mathrm{E}$ ) resulted in the generation of a population that was over $82 \% \mathrm{MMP}_{\mathrm{p} \text {.R158C }}$ neoantigen specific, and capable of recognizing 4177-TMG1 RNA, encoding MMP14 $_{\text {p.R158C }}$ 25-mer (Figure 3F). Hence, in the 2 patients with an available tumor cell line, circulating neoantigen-specific T cells and/ or PBLs expressing the neoantigen-specific TCR recognized their corresponding neoepitope naturally expressed, processed, and presented by the autologous tumor cell line.

Despite the data demonstrating recognition of the autologous tumor cell lines by the enriched populations of neoantigen-specific lymphocytes, $T$ cell responses based on IFN- $\gamma$ ELISPOT and $4-1 \mathrm{BB}$ upregulation appeared weaker compared with that previously reported in melanoma patients (19). When studying the functionality of MMP14 mutation-specific lymphocytes isolated from peripheral blood of NCI-4177 in more detail, we found that only a small percentage of the cells were capable of producing IFN- $\gamma$, upregulating 4-1BB, and degranulating based on CD107a detection (Figure 3G). This frequency increased upon preincubation of the tumor cell line with IFN- $\gamma$, and tumor recognition was 
inhibited by the MHC-I blocking antibody W6/32, demonstrating, once again, antigen-specific recognition of the tumor cells. However, the ability of these cells to kill the autologous tumor was limited when an effector-to-target ratio at which control melanoma TILs effectively killed their autologous tumor was used (not shown), even when the tumor was preincubated with IFN- $\gamma$ (Figure $3 \mathrm{H})$. Importantly, tumor recognition and lysis were enhanced when the autologous tumor was pulsed with the mutated peptide, but not when it was pulsed with the WT counterpart (Figure 3, G and $\mathrm{H}$ ), evidencing that this deficiency was at least in part due to limited processing and presentation of the endogenous neoantigen by the tumor cells. Although the weak in vitro antitumor activity of neoantigen-specific lymphocytes observed in this patient cannot be extended to all neoantigen-specific lymphocytes from GI cancer patients, defective antigen presentation has previously been described in GI tumors (24), and overcoming it could be important for the clinical activity of personalized $\mathrm{T}$ cell therapies targeting neoantigens in patients with GI cancers.

\section{Discussion}

In this study, we investigated the existence of circulating neoantigen-reactive $\mathrm{T}$ cell responses in 7 patients with GI cancers with a mutation burden ranging from 32 to 151 nonsynonymous mutations. Despite their limited mutation burden, the variable and relatively poor TIL infiltration in the corresponding tumor biopsies (Supplemental Table 4), and the largely refractory nature of these cancers to immune checkpoint inhibitors, selection of $\mathrm{CD} 8^{+} \mathrm{PD}-1^{+}, \mathrm{CD} 8^{+} \mathrm{PD}-1^{\text {hi }}$, and $\mathrm{CD} 4^{+} \mathrm{PD}-1^{\text {hi }}$ coupled with a personalized high-throughput screening approach was able to identify and enrich both $\mathrm{CD}^{+}$and/or $\mathrm{CD}^{+}$neoantigen reactivities in the blood of 6 of 7 patients. These results are consistent with the identification of tumor-resident neoantigen-specific lymphocytes in approximately $80 \%$ of patients with GI cancer (13). Hence, this strategy provides a minimally invasive alternative to identify these neoantigen-specific lymphocytes.

Except for KRAS $_{\text {p.G12D }}$, the remaining 9 neoantigens were private neoantigens, and have not been previously detected in other tumors. Given that targeting of neoantigens expressed by all tumor cells could limit the outgrowth of antigen escape tumor variants, we analyzed the intratumoral heterogeneity of the nonsynonymous mutations and neoantigens identified within each patient's tumor $(n=5)$. Analysis of the intratumoral heterogeneity of the nonsynonymous mutations identified for each of the tumors with available exome sequencing data revealed clonal and subclonal mutations within each metastatic tumor deposit (Supplemental Figure 7). Remarkably, all 10 neoantigens targeted by lymphocytes derived from the circulating $\mathrm{CD} 8^{+} \mathrm{PD}-1^{+}, \mathrm{CD} 8^{+} \mathrm{PD}-1^{\text {hi }}$, or $\mathrm{CD} 4^{+} \mathrm{PD}-1^{\text {hi }}$ were clonal. Moreover, we also studied the intertumoral neoantigen heterogeneity in patients NCI-4095 and NCI-4110, for which we sequenced 3 and 2 independent tumor lesions, respectively. KRAS $_{\text {p.G12D }}$ was found to be clonal in all 3 lesions sequenced, 40951, 4095-2A, and 4095-2B, consistent with it being an early genetic alteration driving tumor development. However, DNAJB1 ${ }_{\mathrm{p} . \mathrm{Q} 336 \mathrm{R}}$, targeted by circulating lymphocytes from NCI-4110, was unique and clonal in lesion 4110-A but was not detected in tumor 4110-B, resected and sequenced in parallel. Therefore, a diverse personalized $\mathrm{T}$ cell product targeting multiple neoantigens would be opti- mal to account for neoantigen heterogeneity and other previously reported mechanisms of immune escape $(15,25)$.

A few previous reports have identified neoantigen-specific lymphocytes in peripheral blood of a small number of patients with GI cancer as well as other epithelial cancers with relatively low mutation burden. These relied on successive rounds of in vitro stimulation of either PBMCs or peripheral blood memory $\mathrm{T}$ cells with selected candidate neoantigens or minimal epitopes selected using in silico peptide HLA binding prediction, or relied on the establishment of thousands of $\mathrm{T}$ cell clones for a single patient, which were screened for recognition of all the candidate mutated peptides identified. Our strategy to identify circulating neoantigen-specific lymphocytes differs from those in that we select $\mathrm{T}$ cells expressing the PD- 1 inhibitory receptor, typically expressed on acutely and chronically stimulated lymphocytes. Selection of PD- $1^{+}$or PD- $1^{\text {hi }}$ lymphocytes overcomes the need of serially stimulating the cells in vitro with candidate neoantigens. More importantly, it results in preferential detection of reactivities that are naturally expanded in the patients' blood. Several reports in tumors with high mutation burden, including our previous work in melanoma, previously demonstrated that tumor-resident $\mathrm{CD} 8^{+} \mathrm{PD}-1^{+}$or $\mathrm{CD} 8^{+} \mathrm{PD}-1^{\text {hi }}$ cells contain the vast majority of autologous tumor-reactive lymphocytes (20, 26, 27). Moreover, we demonstrated that the circulating $\mathrm{CD}^{+}$lymphocytes expressing PD-1 were enriched in tumor-reactive and neoantigen-specific lymphocytes in 4 melanoma patients. Consistent with the recognition of autologous tumor observed by the ex vivo-expanded tumor-resident and circulating $\mathrm{CD} 8^{+} \mathrm{PD}-1^{+}$lymphocytes, we found a high degree of overlap between the neoantigens recognized and the composition of the TCRB repertoire of the 2 populations (19). Our current data now demonstrate that this novel approach can enrich for circulating neoantigen-specific lymphocytes by selecting $\mathrm{PD}-1^{+}$and or PD- ${ }^{\text {hi }}$ lymphocytes in patients with low mutation burden, making these findings more widely applicable.

A recent publication raised concerns regarding the use of blood as a source to identify neoantigen-specific lymphocytes (18). The neoantigens targeted by in vitro-sensitized PBMCs from ovarian cancer patients differed from those targeted by TILs, and the neoantigen-specific lymphocytes isolated from peripheral blood displayed lower avidity. Our findings demonstrate that the blood is an attractive alternative source to identify neoantigen reactivities. Five of the 6 neoantigen-specific clonotypes identified in the blood of 3 GI patients were detected at a higher frequency at the tumor site compared with the blood, consistent with local clonal expansion. With the exception of patient NCI-4110, the neoepitopes targeted by peripheral blood $\mathrm{CD}^{+}{ }^{+} \mathrm{PD}-1^{+/ h i} \mathrm{~T}$ cells in NCI-4078, NCI-4095, and NCI-4166 were also recognized by TILs expanded in high-dose IL-2 or by ex vivo-expanded CD8 ${ }^{+}$PD- $1^{+}$TILs (Supplemental Figure 8). Moreover, identical TCRs were identified in both the tumor and blood of GI cancer patients. Such was the case of the KRAS ${ }_{\text {p.G12D }}$-specific TCR- $\alpha \beta$ clonotype that we identified in the circulating CD8 ${ }^{+} \mathrm{PD}-1^{+}$ lymphocytes before cell transfer in patient NCI-4095. This clonotype was identical to 1 of the 4 clonotypes administered to patient NCI-4095, which induced tumor regression (15). Interestingly, the 3 additional KRAS ${ }_{\text {p.G12D }}$ clonotypes identified from ex vivoexpanded TILs, which were not detected in the circulating PD-1+ 
or PD-1 ${ }^{\text {hi }}$ subsets, were far less dominant in the 3 tumor deposits analyzed (Supplemental Table 3). It is tempting to speculate that their limited frequency in the tumor could explain a lower frequency and lack of detection within the peripheral blood CD $8{ }^{+} \mathrm{PD}-1^{+}$cells. In addition to this correlative evidence showing identical neoantigen reactivities between peripheral blood and tumor-resident lymphocytes, we were able to generate tumor cell lines for 2 of the patients studied and found that circulating CD $8{ }^{+} \mathrm{PD}-1^{+/ h i}$ lymphocytes were capable of recognizing their neoepitope when naturally processed and presented by the autologous tumor cell line.

The observation that most patients with DNA mismatch repair-proficient tumors likely harbor both tumor-infiltrating and circulating $\mathrm{T}$ cells targeting the same neoantigens yet generally do not benefit from immune checkpoint blockade therapy highlights the need to better understand the mechanisms behind the lack of efficacy. The limited frequency of some of these neoantigen-specific clonotype cells at the tumor site, as detected with TCR deep sequencing, is in line with a recent publication showing that the frequency of tumor-reactive lymphocytes is variable and rare in epithelial cancers compared with melanoma tumors (11). It is worth noting that we consistently observed lower levels of IFN- $\gamma$ spots and 4-1BB upregulation following coculture of the enriched populations of neoantigen-reactive lymphocytes and PBLs transduced with neoantigen-specific TCRs with the autologous tumor cell lines derived from GI cancer patients, as compared with those we observed previously when performing similar experiments in melanoma patients $(19,20)$. In addition, direct recognition of the tumor cell line by the $\mathrm{CD} 8^{+} \mathrm{PD}-1^{+}$ or PD-1 ${ }^{\text {hi }}$ lymphocytes was marginal, but reproducible (Figure 3 , $\mathrm{E}$ and $\mathrm{F}$ ), compared with that observed for melanoma patients (19). Moreover, MMP14 mutation-specific lymphocytes isolated from the peripheral blood of NCI-4177 displayed weak tumor recognition and cytolytic activity, which were enhanced when the cell line was pulsed with the specific neoepitope recognized. We were unable to investigate whether this phenomenon was present in patient NCI-4110 because of a limitation in T cells. The mechanisms behind the weak tumor reactivity in NCI-4177 are unknown, but our observations are consistent with deficiencies in tumor antigen processing and presentation that have been observed in a variety of cancers (24) and could limit the cytolytic potential of neoantigen-specific lymphocytes in some patients. In such cases, exploiting $\mathrm{CD}^{+} \mathrm{T}$ cell responses or the innate immune system may be necessary to induce antitumor responses. Nonetheless, case reports demonstrating that the transfer of neoantigen-reactive $\mathrm{T}$ cells can mediate antitumor activity in a patient with cholangiocarcinoma and colorectal cancer combined with the progress in the nonviral delivery of TCRs into PBMCs (28) have spurred interest in harnessing personalized $\mathrm{T}$ cells and TCRs derived from the tumor or peripheral blood to treat epithelial cancers. Whether such personalized T cell therapies can reproducibly induce tumor regression in a large cohort of patients with epithelial cancers is unclear and is currently being tested in a clinical trial (NCI-18-C-0049).

The functional implications of generating enriched populations of neoantigen-specific lymphocytes for patient treatment from circulating PD-1-expressing lymphocytes remain unclear.
We did not observe any consistent differences in the ex vivo expansion between the bulk, PD- $1^{-}, \mathrm{PD}-1^{+}$, and PD $-1^{\text {hi }} \mathrm{CD} 8^{+}$and $\mathrm{CD} 4^{+}$lymphocytes isolated from the peripheral blood. Moreover, detailed tracking of the expansion of enriched populations of neoantigen-specific lymphocytes derived from $\mathrm{CD} 8^{+} \mathrm{PD}-1^{+}$, $\mathrm{CD} 8^{+} \mathrm{PD}-1^{\mathrm{hi}}$, or $\mathrm{CD} 4^{+} \mathrm{PD}-1^{\text {hi }}$ (Supplemental Table 5) supports the proliferative potential of these $\mathrm{T}$ cells. The vast majority of the expanded $\mathrm{T}$ cells displayed effector memory markers and expressed similar markers of activation/exhaustion, with the exception of PD-1 expression, which remained slightly increased on the $\mathrm{PD}-1^{+}$-derived and $\mathrm{PD}-1^{\mathrm{hi}}$-derived $\mathrm{CD} 8^{+}$and $\mathrm{CD}^{+}$lymphocytes (Supplemental Figure 9). The partial maintenance of PD-1 is consistent with the possibility that chronic antigen stimulation can lead to epigenetic changes in $\mathrm{T}$ cells $(29,30)$, and the implications of the expression of PD-1 for the clinical efficacy of PD-1-derived neoantigen-specific lymphocytes warrant further investigation. Notably, the infusion products of ex vivo-expanded melanoma TILs frequently display moderate to high levels of PD-1 expression (not shown), and they can induce antitumor responses in approximately $50 \%$ of metastatic melanoma patients. Nonetheless, combination with anti-PD-1 blocking antibodies or the use of PBLs expressing neoantigen-specific TCRs isolated from the blood could overcome potential limitations of using the naturally occurring neoantigen-specific lymphocytes.

Contrary to the extensive analysis of $\mathrm{PD}-1$ expression on $\mathrm{CD} 8^{+}$ $\mathrm{T}$ cells in the setting of cancer, the role of PD- 1 expression on $\mathrm{CD} 4^{+}$ lymphocytes has been far less explored. In healthy mice, PD- 1 is an early activation marker of $\mathrm{CD} 4^{+}$lymphocytes, but its expression is maintained within follicular $\mathrm{T}$ helper $\left(\mathrm{T}_{\mathrm{FH}}\right)$ lymphocytes, specialized in providing help to $\mathrm{B}$ cell responses $(31,32)$. The significance of $\mathrm{PD}-1$ expression and the presence of $\mathrm{T}_{\mathrm{FH}}$ cells at the tumor site or in the blood of patients with cancer remain controversial. A subset of CD $4^{+}$FoxP3-PD- $1^{\text {hi }}$ TILs displaying extensive transcriptional overlap with $\mathrm{T}_{\mathrm{FH}}$ cells was recently reported to be negatively associated with prognosis following anti-PD-1 therapy (33). $\mathrm{T}_{\mathrm{FH}}$ gene signatures, on the other hand, have been associated with a prolonged survival $(34,35)$. Our results show that neoantigen-specific $\mathrm{CD} 4^{+}$cells can express high levels of PD- 1 in peripheral blood, and this can be exploited to enrich and select for neoantigen-specific $\mathrm{CD}^{+}$lymphocytes. Thus, the noninvasive personalized strategy to enrich and detect circulating neoantigen-specific lymphocytes based on selection of PD-1-expressing lymphocytes can be extended to tumors with fewer somatic mutations, such as GI cancers, and can also be applied to enrich and isolate for $\mathrm{CD}^{+}$neoantigen-specific lymphocytes and TCRs, making it a more general and applicable finding.

Our data demonstrate that selection of peripheral blood PD-1-expressing $\mathrm{CD}^{+}$and $\mathrm{CD} 8^{+}$lymphocytes combined with a personalized screening approach can frequently identify neoantigen-reactive lymphocytes circulating in the blood of patients with GI cancer, making this strategy more widely applicable. Consistent with the TCR stimulation-driven expression of PD-1 on neoantigen-reactive lymphocytes, this strategy is particularly attractive to guide the identification of circulating neoantigen-reactive lymphocytes or TCRs that could be used to develop personalized immunotherapies targeting neoantigens. 


\section{Methods}

Patient characteristics, leukapheresis, and tumor samples. Patients NCI4078, NCI-4095, NCI-4110, NCI-4114, and NCI-4177 were chosen for this study on the basis of availability of pretreatment leukapheresis and frozen fresh tumor to perform WES and transcriptome analysis. Pretreatment leukapheresis was available for patients NCI-4166 and NCI-MM, but archive formalin-fixed paraffin-embedded tumor was used as the source to identify nonsynonymous mutations. All the patients included in this study had metastatic disease and had received a wide range of prior therapies, but all were immunotherapy naive. Patient characteristics are summarized in Supplemental Table 1. PBMCs were obtained from a leukapheresis using a Ficoll-Hypaque gradient and cryopreserved until analysis. After surgical resection, tumor specimens were cut into small 2- to 4-mm fragments and either snap-frozen or included in OCT compound (Sakura Finetek) to estimate the percentage of tumor and to extract DNA and RNA. The remaining tissue was minced under sterile conditions, followed by enzymatic digestions for 1.5 hour at $37^{\circ} \mathrm{C}$ and intermittent mechanical tissue separation using gentleMACS (Miltenyi Biotec), and single-cell suspensions were washed and cryopreserved until further analysis.

Exome sequencing, RNA sequencing, and mutation clustering. Genomic DNA and total RNA were purified from fresh tumor biopsy or 10- $\mu \mathrm{m}$ OCT sections and normal PBMCs. WES library construction was performed by exome capture of approximately 20,000 coding genes using SureSelectXT Target Enrichment System (Agilent Technologies), and paired-end sequencing was performed with an Illumina High-Output flow cell kit (300 cycles) on a NextSeq 500 sequencer (Illumina) at the Surgery Branch, National Cancer Institute. The average sequencing depth ranged from 100 to 150 for each of the individual libraries generated. Alignments of WES to the reference human genome build hg19 were performed using NovoAlign MPI from Novocraft (http://www.novocraft.com/). Duplicates were marked using Picard's MarkDuplicates tool (https://github.com/broadinstitute/ picard/releases/tag/1.127). Insertion and deletion (indel) realignment and base recalibration were performed according to Genome Analysis Toolkit (GATK) best practices (https://www.broadinstitute.org/ gatk/). SAMtools was used to create tumor and normal pileup files. Four independent mutation callers (Varscan, SomaticSniper, Mutect, and Strelka) were used to call somatic nonsynonymous mutations (summarized in Table 1) according to the following criteria: minimum coverage of 10 reads, minimum 4 variant reads, greater than $7 \%$ variant allele frequency (VAF) and called by 2 or more callers (single nucleotide variants) or 1 for insertions and deletions, and annotated as coding mutation in 2 of the 3 annotation data sets (RefGene, University of California, Santa Cruz, Ensembl). An mRNA sequencing library was also prepared from tumor biopsies using an Illumina TruSeq RNA library prep kit. RNA alignment was performed using STAR (36). Duplicates were marked using Picard's MarkDuplicates tools. Fragments per million mapped reads (FPKM) values were calculated using Cufflinks (37). The levels of specific transcripts encoding putative NSM variants were calculated as FPKM and used to assess expression of candidate mutations. Tumor from NCI-MM was sequenced and analyzed externally, and the specific filters used to identify the NSM mutations are unknown.

Nonsynonymous mutations for immunological screening were selected based on the presence in both the tumor exome and transcriptome (NCI-4078, NCI-4095) or based on exome only (NCI-4110,
NCI-4177, NCI-4114, NCI-4166, NCI-MM). For patients NCI-4095 and NCI-4110, we included mutations with minimum VAF of $10 \%$ and minimum variant reads of 3 and considered variants with normal coverage less than 10. Mutations were manually vetted using Integrative Genomics Viewer. The mutated 25-mers screened for each of the patients are shown in Supplemental Table 6. With the exception of the raw FASTQ files from NCI-MM, all raw FASTQ files can be accessed through the NCBI database of Genotypes and Phenotypes (dbGaP) using accession phs001003.v1.p1. Whole-exome sequencing of NCI-MM was performed before this patient entered the study, and the raw FASTQ files were analyzed externally but were not available for reanalysis.

Determination of allele-specific copy number analysis and mutation clustering. WES data were used in the copy number analysis. The data analysis was performed in R, version 3.4.0. The segmented copy number, cellularity, and ploidy were determined using Sequenza version 2.1.2 with normal sample as references and hg19 coordinates. The cancer cell fraction (CCF) of each mutation was estimated by integration of the local copy number, tumor purity (obtained from Sequenza), and VAF. Only mutations with tumor coverage of at least 20 times were included in the analysis. The observed mutation copy number can be calculated by integration of local copy number, purity, and VAF. The mutations were classified as either clonal or subclonal based on the confidence interval of the CCF. Mutations were defined as clonal if the $95 \%$ confidence interval overlapped 1, and subclonal otherwise. All mutations with read depth greater than 4 and VAF greater than $7 \%$ were clustered using PyClone version 1.3.0 Dirichlet process clustering. PyClone allows clustering to simply group clonal and subclonal mutations based on their CCF estimates. PyClone was run using 50,000 iterations and a burn-in of 1000 .

Antibodies, flow cytometry, cell sorting, and in vitro expansion of lymphocytes. We purchased fluorescently labeled human-specific antibodies from BD Biosciences (CD3-APC-H7, clone sk7; CD3-APC-Cy7, clone sk7; CD137-APC, clone 4B4-1; CD4-PE, clone RPA-T4; CD8-PE-Cy7, clone sk1; CD134-FITC, clone ACT35), eBioscience (anti-mouse TCR $\beta$ chain [anti-mTCRB]-FITC, clone H57-597), and BioLegend (IFN- $\gamma$ FITC, clone 4S.B3; CD107a-APC-Cy7, clone H4A3; CD27-FITC, clone O323; CD45RO-PE-Cy5, clone UCHL1; CCR7-BV650, clone G043H7; ICOS-BV605, clone C398.4A; CD4-BV510, clone SK3; CD39-BV421, clone A1). Anti-PD-1 (pembrolizumab) was custom conjugated with PE by Miltenyi Biotec in good manufacturing practice (GMP) conditions according to the company's approved standard operating procedures for generating fluorochrome-conjugated antibodies. Anti-PD-1 antibody used for analysis and sorting was a gift from L. Liu (MedImmune; clone AMP-514, 1:300, PD-1 Alexa Fluor 647). Analytical flow cytometry data were acquired on a Canto II cell analyzer (BD Biosciences). All cell sorting experiments were performed using a BD FACSJazz (BD Biosciences). Data were analyzed with FlowJo software (Becton, Dickinson and Co.).

To perform cell sorting from PBMCs, $1 \times 10^{8}$ to $3 \times 10^{8}$ PBMCs were thawed into T cell medium containing DNase (Genentech Inc.; $1: 1000)$, centrifuged, plated at $2 \times 10^{6}$ cells per well in a 24 -well plate in the absence of cytokines, and rested overnight at $37^{\circ} \mathrm{C}$ and $5 \%$ $\mathrm{CO}_{2}$. Cells were harvested, and $\mathrm{CD} 8^{+}$and $\mathrm{CD} 4^{+}$cells were enriched sequentially using CD8 and CD4 microbeads (Miltenyi Biotec) and resuspended in staining buffer. Next, we blocked the Fc receptor using an $\mathrm{Fc}$ receptor blocking antibody (Miltenyi Biotec), counted the cells, resuspended them at $4 \times 10^{7}$ cells $/ \mathrm{mL}$ in staining buffer, and 
incubated them with antibodies for 30 minutes at $4^{\circ} \mathrm{C}$. The pre-enrichment step was not performed when $\mathrm{T}$ cells were sorted from a fresh tumor single-cell suspension. PD-1, $\mathrm{PD}-1^{+}$, and $\mathrm{PD}-\mathrm{1}^{\text {hi }}$ cells were sorted after gating on live $\mathrm{CD} 3^{+} \mathrm{CD} 4^{+}$or $\mathrm{CD} 3^{+} \mathrm{CD} 8^{+}$cells. T cells obtained ranged from 6 cells to $1.5 \times 10^{5}$ cells. When more than $1 \times 10^{4}$ cells were sorted, half of the cells were snap-frozen to perform deep sequencing of the variable V-D-J region of the TRB gene, and the other half was expanded in vitro.

For flow cytometry-based sorting of antigen-reactive lymphocytes, $\mathrm{T}$ cells were selected based on 4-1BB expression following 20 hours of coculture with tumor cells or with antigen-expressing (peptide-pulsed or electroporated) autologous APCs. Cocultured cells were pelleted, resuspended in staining buffer, and incubated with antibodies for 30 minutes at $4^{\circ} \mathrm{C}$. Live $\mathrm{CD}^{+} \mathrm{CD}^{+} 4-1 \mathrm{BB}^{+}$or $\mathrm{CD}^{+} \mathrm{CD}^{+} 4-1 \mathrm{BB}^{+}$ lymphocytes were either bulk-sorted and expanded in vitro, or single cells were sorted into a 96-well plate to perform single-cell, targeted $T R A$ and TRB sequencing.

Sorted cells were expanded using a rapid expansion protocol (REP) consisting of 200-fold excess of irradiated (5000 rad) allogeneic feeders from 3 donors in T cell medium containing $30 \mathrm{ng} / \mathrm{mL}$ anti-CD3 antibody OKT3 (Miltenyi Biotec) and $3000 \mathrm{IU} / \mathrm{mL}$ IL-2 (Chiron). After day 7, medium was replaced every 2 days with fresh $\mathrm{T}$ cell medium containing IL-2. Cells were split when confluent or when they reached a concentration exceeding $3 \times 10^{6}$ cells $/ \mathrm{mL}$. At day 14 , cells were harvested and either used in coculture experiments or cryopreserved until further analysis.

The generation of enriched populations of neoantigen-specific lymphocytes required 2 consecutive stimulations/expansions: (a) stimulation of sorted PD-1+ $1^{+}$or PD-1 ${ }^{\text {hi }}$ cells derived from PBMCs, and (b) stimulation of $4-1 \mathrm{BB}^{+}$sorted lymphocytes after coculture of the PD $-1^{+}$and/or PD- $1^{\text {hi }}$ cells against the peptide-pulsed autologous APCs.

We evaluated the expansion of $4-1 \mathrm{BB}^{+}$sorted cells after coculture of the $\mathrm{CD} 8^{+} \mathrm{PD}-1^{+}, \mathrm{CD} 8^{+} \mathrm{PD}-1^{\mathrm{hi}}$, or $\mathrm{CD} 4^{+} \mathrm{PD}-1^{\text {hi }}$ subsets, which had already undergone 1 REP, from 2 randomly selected patients (NCI-4095 and NCI-4078) against some representative neoantigens (KRAS ${ }_{\text {p.G12D }}$, PSMD2 ${ }_{\text {p.G644A }}$, and DLAT $\left.{ }_{\text {p.G294L }}\right)$. The expansion of these populations at day 14 after stimulation ranged from 7000- to 94,000-fold (Supplemental Table 5). The proliferative potential of these cells suggests that hundreds of millions to billions of enriched neoantigen-specific lymphocytes can be generated for patient treatment starting from tens of thousands of $\mathrm{PD}-1^{+} \mathrm{T}$ cells sorted from peripheral blood.

Cytotoxicity assay. CFSE-labeled tumor cells $\left(3 \times 10^{4}\right)$ were cocultured with $\mathrm{T}$ cells at an effector-to-target ratio of 2 (Pher4177 CD8 ${ }^{+}$ PD- $1^{\text {hi }}$ TMG1 enriched) or 5 (Pher4177 CD8 ${ }^{+}$PD- $1^{\text {hi }}$ TCL enriched) for 20 hours. Tumor cells alone were used as negative control, and staurosporine-treated cells $(10 \mu \mathrm{M})$ were used as positive controls of cell death. For MHC-I blocking, CFSE-labeled tumor cells were preincubated with $200 \mu \mathrm{g} / \mathrm{mL}$ anti-MHC-I antibody (clone W6/32) for 1 hour, and blocking antibody was maintained during coculture at $50 \mu \mathrm{g} / \mathrm{mL}$. After 20 hours of coculture, cells were trypsinized and stained with LIVE/ DEAD Violet fixable stain (Thermo Fisher Scientific) for 30 minutes at room temperature. The percentage of dead tumor cells (i.e., $\mathrm{CFSE}^{+}$and LIVE/DEAD Violet ${ }^{+}$) was determined by flow cytometry, and specific lysis was calculated from duplicates as (\% experimental death - \% basal death) / (\% maximal staurosporine death - \% basal death).

Generation of APCs. Monocyte-derived immature DCs were generated using the plastic adherence method. Briefly, PBMCs isolated from a leukapheresis were thawed, washed, and incubated for 90 minutes at $37^{\circ} \mathrm{C}$ and $5 \% \mathrm{CO}_{2}$ in neat AIM-V medium (Thermo Fisher Scientific) in a tissue culture-treated flask at approximately $1 \times 10^{6}$ cells $/ \mathrm{cm}^{2}$. After this period, nonadherent cells were removed by vigorous washing of the flasks with AIM-V, and then incubated again for 60 minutes in AIM-V medium. Nonadherent cells were washed, and adherent cells were incubated with DC medium. On day 3, fresh DC medium was added, and on day 5 to 6 , immature DCs were harvested. Fresh or cryopreserved DCs were used in the experiments. When used after cryopreservation, cells were thawed into DC medium, spun at $1000 \mathrm{rpm}$ for 10 minutes, resuspended in DC medium at $2 \times 10^{6}$ cells $/ \mathrm{mL}$, and incubated at $37^{\circ} \mathrm{C}$ and $5 \% \mathrm{CO}_{2}$ for 2 hours before peptide pulsing or electroporation.

Antigen-presenting B cell lines were generated by isolation of B cells from $\mathrm{PBMCs}$ by positive selection using $\mathrm{CD} 19^{+}$microbeads (Miltenyi Biotec). B cells were expanded in B cell medium using irradiated NIH 3T3 CD4OL cells. At day 3, fresh B cell medium was replenished. B cells were used fresh or cryopreserved at day 5 to 6 . When used after cryopreservation, cells were thawed into B cell medium the day before electroporation.

Peptide synthesis and peptide pulsing. We purchased peptides from GenScript or JPT. Crude peptides were used for the initial in vitro screening of $\mathrm{T}$ cells. To validate reactivities observed in the initial screen, we ordered selected HPLC-purified mutant peptides and their WT counterparts. APCs (DCs or B cells) were harvested, washed, and resuspended at $2 \times 10^{6}$ to $5 \times 10^{6}$ cells $/ \mathrm{mL}$ in their corresponding media supplemented with the appropriate cytokines with $10 \mu \mathrm{g} / \mathrm{mL}$ or $1 \mu \mathrm{g} /$ $\mathrm{mL}$ for 25-mers and minimal epitopes, respectively. Pulsing of peptide pools was performed at a final concentration of $10 \mu \mathrm{g} / \mathrm{mL}$ per peptide. After overnight pulsing, APCs were washed with PBS, resuspended in $\mathrm{T}$ cell medium, and immediately used in coculture assays.

HLA identification and minimal epitope prediction. HLA was determined from the WES data using the PHLAT (38) algorithm. HLA-I restriction elements identified are shown in Supplemental Table 7. Candidate minimal MMP14 ${ }_{\text {p.R158C }}$ epitopes including mutant residues predicted to bind to their autologous HLA-I molecules were identified using NetMHCpan version 4.0 (39). HLA-C ${ }^{*} 08$ :02-restricted WT and mutated KRAS $_{\text {p.G12D }}$ 9-mers and 10-mers were reported previously (15).

$T M G$ construction and in vitro transcription of RNA and electroporation. TMGs were constructed as previously described (17). For each nonsynonymous variant identified by WES, we constructed a "minigene," consisting of the mutant amino acid flanked by 12 amino acids of the WT protein sequence. Up to 17 minigenes were concatenated to generate a TMG construct. TMG constructs were codon optimized and subcloned into pcRNA2SL using EcoRI and BamHI. pcRNA2SL is based on pcDNA3.1, modified to include a signal sequence and a DC-LAMP trafficking sequence, previously described as enhancing processing and presentation (40). Sanger sequencing was used to verify the nucleotide sequence of the TMG constructs. One microgram of linearized (NotI; New England Biolabs) plasmid RNA was used as a template to generate in vitro-transcribed TMG RNA using the Mmessage Mmachine T7 Ultra kit (Life Technologies) as instructed by the manufacturer. RNA was precipitated using $\mathrm{LiCl}_{2}$, and RNA concentration was measured using a NanoDrop spectrophotometer. RNA was resuspended at $1 \mu \mathrm{g} / \mu \mathrm{L}$, aliquoted, and stored at $-80^{\circ} \mathrm{C}$ until use. RNA encoding WT and mutated $\mathrm{KRAS}_{\text {p.G12D }}$ was generated by subcloning of the full-length cDNA into pcRNA2SL and in vitro transcription of RNA, as described above. 
Assessment of target cell recognition: IFN- $\gamma$ ELISPOT assay and detection of 4-1BB, CD107a, and IFN- $\gamma$. T cells were thawed into T cell medium supplemented with 3000 IU IL-2 two days before coincubation with target cells. Before each coculture, target cells and T cells were washed and replaced with cytokine-free $\mathrm{T}$ cell medium. Typically, equal volumes $(100 \mu \mathrm{L})$ of $\mathrm{T}$ cells and APCs or tumor cells were mixed together in a 96 -well plate. $2 \times 10^{4}$ to $5 \times 10^{4} \mathrm{~T}$ cells were coincubated with $1 \times 10^{5}$ or $3 \times 10^{5}$ peptide-pulsed or TMG-electroporated DCs or B cells, respectively. Alternatively, T cells were cocultured with $1 \times 10^{5}$ tumor cells. All cocultures were performed in the absence of exogenously added cytokines. For all the assays, plate-bound OKT3 $(1 \mu \mathrm{g} / \mathrm{mL}$; Miltenyi Biotec) was used as a positive control. Media, DMSO (Sigma-Aldrich), and/or irrelevant peptides were used as negative controls.

Cell surface $\mathrm{T}$ cell activation receptors OX40 and 4-1BB were assessed by flow cytometry at approximately 20 hours after stimulation. Briefly, cocultured cells were pelleted, resuspended in staining buffer, and incubated with antibodies for 30 minutes at $4^{\circ} \mathrm{C}$. Cells were washed and resuspended in staining buffer containing propidium iodide (Sigma-Aldrich) and acquired on a BD LSRFortessa flow cytometer. For MHC-I blocking, CFSE-labeled tumor cells were preincubated with $200 \mu \mathrm{g} / \mathrm{mL}$ anti-MHC-I antibody for 1 hour, and blocking antibody was maintained during coculture at $50 \mu \mathrm{g} / \mathrm{mL}$. Anti-MHC-I clone W6/32 hybridoma was produced and purified by LEITAT, according to its approved standard production and purification procedures.

$\mathrm{CD} 107$ and IFN- $\gamma$ were detected at 6 hours after stimulation. Briefly, antibodies specific for the degranulation marker CD107a were added at the beginning of the coculture. Cells were cocultured with target cells in the presence of $5 \mu \mathrm{g} / \mathrm{mL}$ brefeldin A (Sigma-Aldrich) and $2 \mu \mathrm{M}$ monensin (BioLegend). At 6 hours after stimulation, cells were stained for cell surface receptors and then fixed and permeabilized using the Cytofix/Cytoperm kit (BD Biosciences) according to the manufacturer's instructions. Cells were stained with anti-human IFN- $\gamma$ antibody, washed, and acquired on a BD LSRFortessa flow cytometer. Data were analyzed using FlowJo software (Becton, Dickinson and Co.).

Generation of tumor cell lines. Three- to four-millimeter fragments derived from a fresh tumor specimen or $1 \times 10^{6}$ cells from a single cell suspension were cultured in RPMI 1640 supplemented with $20 \%$ Hyclone FBS (GE Healthcare), $100 \mathrm{U} / \mathrm{mL}$ penicillin, $100 \mu \mathrm{g} / \mathrm{mL}$ streptomycin, $25 \mathrm{mM}$ HEPES (Thermo Fisher Scientific), at $37^{\circ} \mathrm{C}$ in $5 \% \mathrm{CO}_{2}$. Medium was replaced once every month until cell lines were established. Once established, the colorectal and pancreatic cell lines TC4110 and TC4177 were sequenced and cryopreserved at early passage. NIH 3T3 CD4OL cells were established by transduction of NIH 3T3 cells (American Type Culture Collection) with a retrovirus encoding CD4OL. NIH 3T3 CD4OL cells were maintained in DMEM supplemented with $10 \%$ Hyclone FBS, $100 \mathrm{U} / \mathrm{mL}$ penicillin, $100 \mu \mathrm{g} / \mathrm{mL}$ streptomycin, $25 \mathrm{mM}$ HEPES (Thermo Fisher Scientific), at $37^{\circ} \mathrm{C}$ in $5 \% \mathrm{CO}_{2}$.

TCR sequencing and construction. High-throughput TRA and TRB targeted sequencing of the variable V-J or V-D-J regions was performed after DNA extraction of pelleted ex vivo-expanded $\mathrm{T}$ cells, sorted T cells, or snap-frozen tumor by Adaptive Biotechnologies. The immunoSEQ platform was used for analysis. Frequencies of specific CDR3 (complementarity-determining region 3) hypervariable amino acid sequences were calculated after exclusion of nonproductive TCR rearrangements.
Single-cell TRBV and TRAV sequencing was performed as previously described (41). Briefly, selected $4-1 \mathrm{BB}^{+}$cells were sorted into reverse transcriptase PCR buffer. We set up a multiplex PCR using a One-Step RT-PCR kit (Qiagen) and multiple V $\alpha$ and V $\beta$ region-specific primers for the reverse transcription and the first amplification. For the second round of amplification we used the first PCR product as a template and set up 2 independent reactions using HotStarTaq DNA polymerase (Qiagen) with either nested $\mathrm{V} \alpha$ and $\mathrm{C} \alpha$ region-specific primers or nested $\mathrm{V} \beta$ and $\mathrm{C} \beta$ region-specific primers. The resulting PCR products were sequenced by the Sanger method with internally nested $C \alpha$ and $C \beta$ region-specific primers, respectively.

$T C R$ construction and transduction of T cells. TRA and TRB CDR3 nucleotide sequence was obtained by next-generation sequencing or by single-cell TCR sequencing, and the $5^{\prime}$ and $3^{\prime}$ ends of the TRBV and TRAV regions were obtained from the International Immunogenetics Information System (IMGT) online database (http://www. imgt.org). The sequence encoding the resulting human TRB V-D-J or TRA V-J was fused to sequences encoding the modified mouse constant TRB chain and TRA, respectively, as previously described (42-44). A gene fragment encoding the codon-optimized full-length $T R B$ and TRA, in this orientation, separated by a furan SGSG P2A linker, was subcloned into pMSGV1 retroviral vector (GenScript).

We generated a transient retroviral supernatant by transfecting the vector encoding the TCR of interest into 293GP cells, provided by S. Feldman (Surgery Branch, National Cancer Institute). PBLs were activated in $\mathrm{T}$ cell medium supplemented with $50 \mathrm{ng} / \mathrm{mL}$ anti-CD3 and $300 \mathrm{IU} / \mathrm{mL}$ IL-2 for 3 days before retroviral transduction. Retroviral supernatants were harvested at 24 and 48 hours, centrifuged to discard cell debris, and diluted 1:1 with medium and used to transduce the activated lymphocytes using the spinoculation method, as previously described (14). Transduced as well as mock-transduced and GFP-transduced lymphocytes, used as controls in all experiments, were used at day 15 after stimulation.

Media for cell culture, and other reagents. T cell medium comprised a 1:1 mix of RPMI 1640 with L-glutamine (Lonza) and AIM-V (Thermo Fisher Scientific) supplemented with $100 \mathrm{U} / \mathrm{mL}$ penicillin, $100 \mu \mathrm{g} /$ $\mathrm{mL}$ streptomycin, $12.5 \mathrm{mM}$ HEPES (Thermo Fisher Scientific), and 5\% human serum (prepared in-house) without cytokines. T cell medium was supplemented with IL-2 as specified in each section. Staining buffer contained PBS supplemented with $0.5 \%$ BSA and 2 mM EDTA (Sigma-Aldrich). DC medium comprised RPMI containing 5\% human AB serum, $100 \mathrm{U} / \mathrm{mL}$ penicillin, $100 \mu \mathrm{g} / \mathrm{mL}$ streptomycin, $2 \mathrm{mM}$ L-glutamine (Thermo Fisher Scientific), $800 \mathrm{IU} / \mathrm{mL}$ GM-CSF, and $200 \mathrm{U} / \mathrm{mL}$ IL-4 (PeproTech). B cell medium comprised IMDM (Quality Biological Inc.) supplemented with $10 \%$ human $\mathrm{AB}$ serum (processed in-house), $100 \mathrm{U} / \mathrm{mL}$ penicillin, $100 \mu \mathrm{g} / \mathrm{mL}$ streptomycin, $2 \mathrm{mM}$ L-glutamine, and $200 \mathrm{U} / \mathrm{mL}$ IL-4. IFN- $\gamma$ was purchased from PeproTech.

Statistics. Data were reported as mean \pm SEM. Experiments were performed without duplicates, unless otherwise specified. All experiments were repeated at least twice. One-way ANOVA and Dunn's test for multiple comparisons were used to test for statistical differences in PD-1 expression on the peripheral blood $\mathrm{T}$ cell subsets after expansion. A $P$ value less than 0.05 was considered statistically significant.

Study approval. Patients were enrolled on a clinical protocol (NCT01174121, ClinicalTrials.gov) approved by the institutional review board of the National Cancer Institute, and signed an informed consent. 


\section{Author contributions}

AG developed the PD-1 selection method, generated the tumor cell lines, and performed the experiments to detect neoantigen-specific $\mathrm{T}$ cells and TCRs, as well as the experiments to evaluate reactivity against the autologous tumor. ET, MRP, SI, RY, and EG performed experiments to detect neoantigen-specific $\mathrm{T}$ cells from the circulating $\mathrm{T}$ cell subsets isolated. AGG and CAF evaluated the proliferative potential, phenotype, and cytotoxicity of neoantigen-specific lymphocytes. AP performed the TCR single-cell sequencing. PFR contributed to minimal peptide prediction and experimental design. TDP performed the DNA and RNA sequencing. JJG carried out the bioinformatics analysis for candidate neoantigen identification and HLA identification. LJ performed the allele-specific copy number analysis and mutation clustering. SR, LN, and JRW aided with the generation of target cells and performed experiments. JCY contributed to experimental design. SAR contributed to experimental design and oversaw the project. All authors discussed the results. AG and SAR wrote the manuscript.

\section{Acknowledgments}

We thank M. Ahmadzadeh, D. Denniger, K. Hanada, and G. Cafri for helpful discussion, and A. Mixon and S. Farid for assistance with cell sorting. This work was supported by the Center for Cancer Research at the National Cancer Institute of the US National Institutes of Health (NIH). AG was supported by Spanish Instituto de Salud Carlos III funding, an initiative of the Spanish Ministry of Economy and Innovation (MS15/00058), the Fero Foundation, and the Cellex Foundation. AG is a member of the BBVA Foundation (FBBVA) Comprehensive Program of Cancer Immunotherapy and Immunology (CAIMI). The work of AG was supported in part by Merck KGaA, Darmstadt, Germany (Grant for Oncology Innovation 2017). CAF was supported by the Asociación Española Contra el Cáncer. Merck KGaA, Darmstadt, Germany, reviewed the manuscript only for medical accuracy before journal submission. The authors are fully responsible for the content of this article, and the views and opinions described in the publication reflect solely those of the authors.

Address correspondence to: Alena Gros, Vall d'Hebron Institute of Oncology (VHIO), Cellex Center, c/Natzaret 115, 08035 Barcelona, Spain. Phone: 34.93.254.34.50 ext. 8700; Email: agros@vhio.net. Or to: Steven A. Rosenberg, Surgery Branch, National Cancer Institute, National Institutes of Health, 10 Center Drive, Bethesda, Maryland 20892, USA. Phone: 240.858.3080; Email: sar@nih.gov.
1. Robbins PF, et al. Mining exomic sequencing data to identify mutated antigens recognized by adoptively transferred tumor-reactive $\mathrm{T}$ cells. Nat Med. 2013;19(6):747-752.

2. van Rooij N, et al. Tumor exome analysis reveals neoantigen-specific $\mathrm{T}$-cell reactivity in an ipilimumab-responsive melanoma. JClin Oncol. 2013;31(32):e439-e442.

3. Lu YC, et al. Mutated PPP1R3B is recognized by T cells used to treat a melanoma patient who experienced a durable complete tumor regression. JImmunol. 2013;190(12):6034-6042.

4. Snyder A, et al. Genetic basis for clinical response to CTLA-4 blockade in melanoma. $N$ Engl J Med. 2014;371(23):2189-2199.

5. Rizvi NA, et al. Cancer immunology. Mutational landscape determines sensitivity to PD-1 blockade in non-small cell lung cancer. Science. 2015;348(6230):124-128.

6. Le DT, et al. PD-1 blockade in tumors with mismatch-repair deficiency. N Engl JMed. 2015;372(26):2509-2520.

7. Le DT, et al. Mismatch repair deficiency predicts response of solid tumors to PD-1 blockade. Science. 2017;357(6349):409-413.

8. Samstein RM, et al. Tumor mutational load predicts survival after immunotherapy across multiple cancer types. Nat Genet. 2019;51(2):202-206

9. Lawrence MS, et al. Mutational heterogeneity in cancer and the search for new cancer-associated genes. Nature. 2013;499(7457):214-218.

10. Vogelstein B, Papadopoulos N, Velculescu VE, Zhou S, Diaz LA, Kinzler KW. Cancer genome landscapes. Science. 2013;339(6127):1546-1558.

11. Scheper W, et al. Low and variable tumor reactivity of the intratumoral TCR repertoire in human cancers. Nat Med. 2019;25(1):89-94.

12. Wang TL, et al. Prevalence of somatic alterations in the colorectal cancer cell genome. Proc Natl
Acad Sci U S A. 2002;99(5):3076-3080.

13. Tran E, et al. Immunogenicity of somatic mutations in human gastrointestinal cancers. Science. 2015;350(6266):1387-1390.

14. Tran E, et al. Cancer immunotherapy based on mutation-specific $\mathrm{CD} 4^{+} \mathrm{T}$ cells in a patient with epithelial cancer. Science. 2014;344(6184):641-645

15. Tran E, et al. T-cell transfer therapy targeting mutant KRAS in cancer. $N$ Engl JMed. 2016;375(23):2255-2262.

16. Cafri G, et al. Memory T cells targeting oncogenic mutations detected in peripheral blood of epithelial cancer patients. Nat Commun. 2019;10(1):449.

17. Martin SD, Wick DA, Nielsen JS, Little N, Holt RA, Nelson BH. A library-based screening method identifies neoantigen-reactive $\mathrm{T}$ cells in peripheral blood prior to relapse of ovarian cancer. Oncoimmunology. 2017;7(1):e1371895.

18. Bobisse S, et al. Sensitive and frequent identification of high avidity neo-epitope specific CD8 ${ }^{+}$ $\mathrm{T}$ cells in immunotherapy-naive ovarian cancer. Nat Commun. 2018;9(1):1092.

19. Gros A, et al. Prospective identification of neoantigen-specific lymphocytes in the peripheral blood of melanoma patients. Nat Med. 2016;22(4):433-438.

20. Gros A, et al. PD-1 identifies the patient-specific $\mathrm{CD}^{+}$tumor-reactive repertoire infiltrating human tumors. J Clin Invest. 2014;124(5):2246-2259.

21. Wolfl M, et al. Activation-induced expression of CD137 permits detection, isolation, and expansion of the full repertoire of $\mathrm{CD} 8^{+} \mathrm{T}$ cells responding to antigen without requiring knowledge of epitope specificities. Blood. 2007;110(1):201-210.

22. Bryant KL, Mancias JD, Kimmelman AC, Der CJ. KRAS: feeding pancreatic cancer proliferation. Trends Biochem Sci. 2014;39(2):91-100.

23. Vaughn CP, Zobell SD, Furtado LV, Baker CL,
Samowitz WS. Frequency of KRAS, BRAF, and NRAS mutations in colorectal cancer. Genes Chromosomes Cancer. 2011;50(5):307-312.

24. Leone P, Shin EC, Perosa F, Vacca A, Dammacco F, Racanelli V. MHC class I antigen processing and presenting machinery: organization, function, and defects in tumor cells. J Natl Cancer Inst. 2013;105(16):1172-1187.

25. McGranahan N, et al. Clonal neoantigens elicit $\mathrm{T}$ cell immunoreactivity and sensitivity to immune checkpoint blockade. Science. 2016;351(6280):1463-1469.

26. Inozume T, et al. Selection of CD8 ${ }^{+} \mathrm{PD}-1^{+}$lymphocytes in fresh human melanomas enriches for tumor-reactive T cells. J Immunother. 2010;33(9):956-964.

27. Thommen DS, et al. A transcriptionally and functionally distinct PD $-1^{+} \mathrm{CD}^{+} \mathrm{T}$ cell pool with predictive potential in non-small-cell lung cancer treated with PD-1 blockade. Nat Med. 2018;24(7):994-1004.

28. Roth TL, et al. Reprogramming human T cell function and specificity with non-viral genome targeting. Nature. 2018;559(7714):405-409.

29. Youngblood B, et al. Chronic virus infection enforces demethylation of the locus that encodes PD-1 in antigen-specific CD8(+) T cells. Immunity. 2011;35(3):400-412.

30. Philip M, et al. Chromatin states define tumour-specific T cell dysfunction and reprogramming. Nature. 2017;545(7655):452-456.

31. Kerfoot SM, et al. Germinal center B cell and T follicular helper cell development initiates in the interfollicular zone. Immunity. 2011;34(6):947-960.

32. Shi J, Hou S, Fang Q, Liu X, Liu X, Qi H. PD-1 controls follicular $\mathrm{T}$ helper cell positioning and function. Immunity. 2018;49(2):264-274.e4.

33. Zappasodi R, et al. Non-conventional inhibitory CD $4^{+}$Foxp $3-\mathrm{PD}-1^{\text {hi }} \mathrm{T}$ cells as a biomarker of 


\section{RESEARCH ARTICLE}

immune checkpoint blockade activity. Cancer Cell. 2018;33(6):1017-1032.e7.

34. Bindea G, et al. Spatiotemporal dynamics of intratumoral immune cells reveal the immune landscape in human cancer. Immunity. 2013;39(4):782-795.

35. Gu-Trantien C, et al. $\mathrm{CD} 4^{+}$follicular helper $\mathrm{T}$ cell infiltration predicts breast cancer survival. J Clin Invest. 2013;123(7):2873-2892.

36. Dobin A, et al. STAR: ultrafast universal RNA-seq aligner. Bioinformatics. 2013;29(1):15-21.

37. Trapnell C, et al. Transcript assembly and quantification by RNA-Seq reveals unannotated transcripts and isoform switching during cell differentiation. Nat Biotechnol. 2010;28(5):511-515.

38. Bai Y, Ni M, Cooper B, Wei Y, Fury W. Inference of high resolution HLA types using genome-wide RNA or DNA sequencing reads. BMC Genomics. 2014;15:325.

39. Jurtz V, Paul S, Andreatta M, Marcatili P, Peters B, Nielsen M. NetMHCpan-4.0: improved peptide-MHC Class I interaction predictions integrating eluted ligand and peptide binding affinity data. JImmunol. 2017;199(9):3360-3368.

40. Wu TC, et al. Engineering an intracellular pathway for major histocompatibility complex class II presentation of antigens. Proc Natl Acad Sci U S A 1995;92(25):11671-11675.

41. Pasetto A, et al. Tumor- and neoantigen-reactive T-cell receptors can be identified based on their frequency in fresh tumor. Cancer Immunol Res. 2016;4(9):734-743.
42. Cohen CJ, Zhao Y, Zheng Z, Rosenberg SA, Morgan RA. Enhanced antitumor activity of murine-human hybrid T-cell receptor (TCR) in human lymphocytes is associated with improved pairing and TCR/CD3 stability. Cancer Res. 2006;66(17):8878-8886.

43. Cohen CJ, Li YF, El-Gamil M, Robbins PF, Rosenberg SA, Morgan RA. Enhanced antitumor activity of $\mathrm{T}$ cells engineered to express $\mathrm{T}$-cell receptors with a second disulfide bond. Cancer Res. 2007;67(8):3898-3903.

44. Haga-Friedman A, Horovitz-Fried M, Cohen CJ. Incorporation of transmembrane hydrophobic mutations in the TCR enhance its surface expression and T cell functional avidity. JImmunol. 2012;188(11):5538-5546. 